\title{
13. QUANTITY, PROVENANCE, AND MATURITY OF ORGANIC MATTER AT ODP SITES 645, 646, AND 647: IMPLICATIONS FOR RECONSTRUCTION OF PALEOENVIRONMENTS IN BAFFIN BAY AND LABRADOR SEA DURING TERTIARY AND QUATERNARY TIME1
}

\author{
Ruediger Stein, ${ }^{2}$ Ralf Littke, ${ }^{3}$ Rainer Stax, ${ }^{2}$ \\ and \\ Dietrich H. Welte ${ }^{3}$
}

\begin{abstract}
The quantity and quality of organic carbon of Eocene to Holocene sediments from ODP Sites 645, 646, and 647 were investigated to reconstruct depositional environments. Results were based on organic-carbon and nitrogen determinations, Rock-Eval pyrolysis, and kerogen microscopy.

The sediments at Site 645 in Baffin Bay are characterized by relatively high organic-carbon values, most of which range from $0.5 \%$ to almost $3 \%$, with maximum values in the middle Miocene. Distinct maxima of organic-carbon accumulation rates occur between 18 and $12.5 \mathrm{Ma}$ and between 3.4 and $0 \mathrm{Ma}$. At Sites 646 and 647 in the Labrador Sea, organic-carbon contents vary between $0.1 \%$ and $0.75 \%$. Cyclic "Milankovitch-type" changes in organic-carbon deposition imply climate-controlled mechanisms that cause these fluctuations.

The composition of organic matter at Site 645 is dominated by terrigenous components throughout the entire sediment sequence. An increased content of marine organic carbon was recorded only in the late-middle Miocene. At Sites 646 and 647 , the origin of the organic matter most probably is marine.

Oceanic paleoproductivity values were estimated, based on the amount of marine organic carbon. During most of the Neogene time interval at Site 645, productivity was low, i.e., similar or less than that measured in Baffin Bay today. Higher values of up to $150(200) \mathrm{gC} \cdot \mathrm{m}^{-2} \cdot \mathrm{y}^{-1}$ may have occurred only in the Miocene. At Sites 646 and 647 , mean paleoproductivity values vary between 90 and $170 \mathrm{gC} \cdot \mathrm{m}^{-2} \cdot \mathrm{y}^{-1}$; i.e., these are also similar to those measured in the Labrador Sea today. Lower values of 40 to $70 \mathrm{gC} \cdot \mathrm{m}^{-2} \cdot \mathrm{y}^{-1}$ were estimated for the early Eocene and (middle) Miocene.
\end{abstract}

\section{INTRODUCTION}

During Leg 105, three sites were drilled in Baffin Bay and the Labrador Sea (Fig. 1). The main objectives of Leg 105 were to study the early tectonic, paleoclimatic, and paleoceanographic evolution of the North Atlantic Ocean and its connection to the Arctic Ocean. Baffin Bay and the Labrador Sea probably were the main connection between the North Atlantic and the Arctic Ocean before the opening of the Greenland and Norwegian seas (Srivastava et al., 1981). Thus, the distinct paleoenvironmental changes that occurred in this region during Tertiary time (such as the tectonic development of new oceanic basins and the onset of glacial climate in the arctic area) should have resulted in distinct changes in sediment composition. A detailed sedimentological, paleontological, and geochemical study of deep-sea sediments from Baffin Bay and the Labrador Sea, therefore, provided a useful tool for reconstructing paleoenvironments of the North Atlantic/Arctic Ocean region.

Site 645 was drilled in Baffin Bay at a position of $70^{\circ} 27.48^{\prime} \mathrm{N}$, $64^{\circ} 39.30^{\prime} \mathrm{W}$ and at a water depth of $2018 \mathrm{~m}$. Total depth of penetration was 1147.1 meters below seafloor (mbsf). Sediments are of early Miocene to Holocene age, predominantly terrigenous clay, silt, and sand; biogenic components are of only minor importance (Fig. 2). Abundant dropstones and other coarse sediments characterize the upper $335 \mathrm{~m}(465 \mathrm{~m})$ of the sediment sequence. Furthermore, for normal marine sediments, these sediments are characterized by unusually high organic-carbon contents, which increased our interest in Site 645 .

\footnotetext{
${ }^{1}$ Srivastava, S., Arthur, M. A., Clement, B., et al., 1989. Proc. ODP, Sci. Results, 105: College Station, TX (Ocean Drilling Program).

2 Institut für Geowissenschaften und Lithosphärenforschung (IGL), Universität Giessen, Giessen, Federal Republic of Germany.

${ }^{3}$ Institut für Erdöl und Organische Geochimie, KFA Jülich GmbH Jülich, Federal Republic of Germany.
}

Site 646 was drilled in a water depth of $3451 \mathrm{~m}$, at $58^{\circ} 12.56^{\prime} \mathrm{N}$, $48^{\circ} 22.15^{\prime} \mathrm{W}$ on the northern flank of the Eirik Ridge, Labrador Sea. Total depth of penetration was 766.7 mbsf. Sediments recovered are of late Miocene to Holocene age (Fig. 2). The lower part of the sequence ( 766.7 to $236.1 \mathrm{mbsf}$ ) mainly consists of greenish-gray clay (claystone) and silt (siltstone), whereas the upper $236.1 \mathrm{~m}$ is characterized by dark gray to light gray clays and silts with carbonate contents of up to $40 \%$ and a common occurrence of dropstones (Fig. 2; Srivastava, Arthur, et al., 1987).

Site 647 was drilled in the Labrador Sea south of the Gloria Drift, in a water depth of $3862 \mathrm{~m}$, at $53^{\circ} 19.88^{\prime} \mathrm{N}, 45^{\circ} 15.72^{\prime} \mathrm{W}$. At Site 647, a 699-m-thick sediment sequence of early Eocene to Holocene age was penetrated. At 699 mbsf, the basaltic basement was reached (Srivastava, Arthur, et al., 1987). The sediments are dominated by terrigenous material in the upper 116 mbsf and again below 530 mbsf. The middle part of the sequence is characterized by biogenic carbonate and siliceous oozes dominated by biogenic silica between 250 and 150 mbsf (Fig. 2; Bohrmann and Stein, this volume).

This study concentrates on the changes in quantity and quality of organic carbon with time. Main interests are the relationships between organic-carbon deposition and both paleoclimatic and paleoceanic conditions in Baffin Bay and the Labrador Sea. Possible changes in thermal maturity of organic matter with increasing depth are also of interest.

\section{MODERN ENVIRONMENT IN BAFFIN BAY AND LABRADOR SEA}

Baffin Bay, located between Greenland and Baffin Island (Fig. 1), is connected to the Arctic Ocean in the north by the narrow Nares Strait and to the Atlantic Ocean in the south by the shallow Davis Strait (less than $600 \mathrm{~m}$ water depth). In the center of Baffin Bay, water depths exceed $2300 \mathrm{~m}$. Surface-water circulation in Baffin Bay is dominated by currents entering the bay from the Arctic Ocean (the Baffin Island Current) and the 


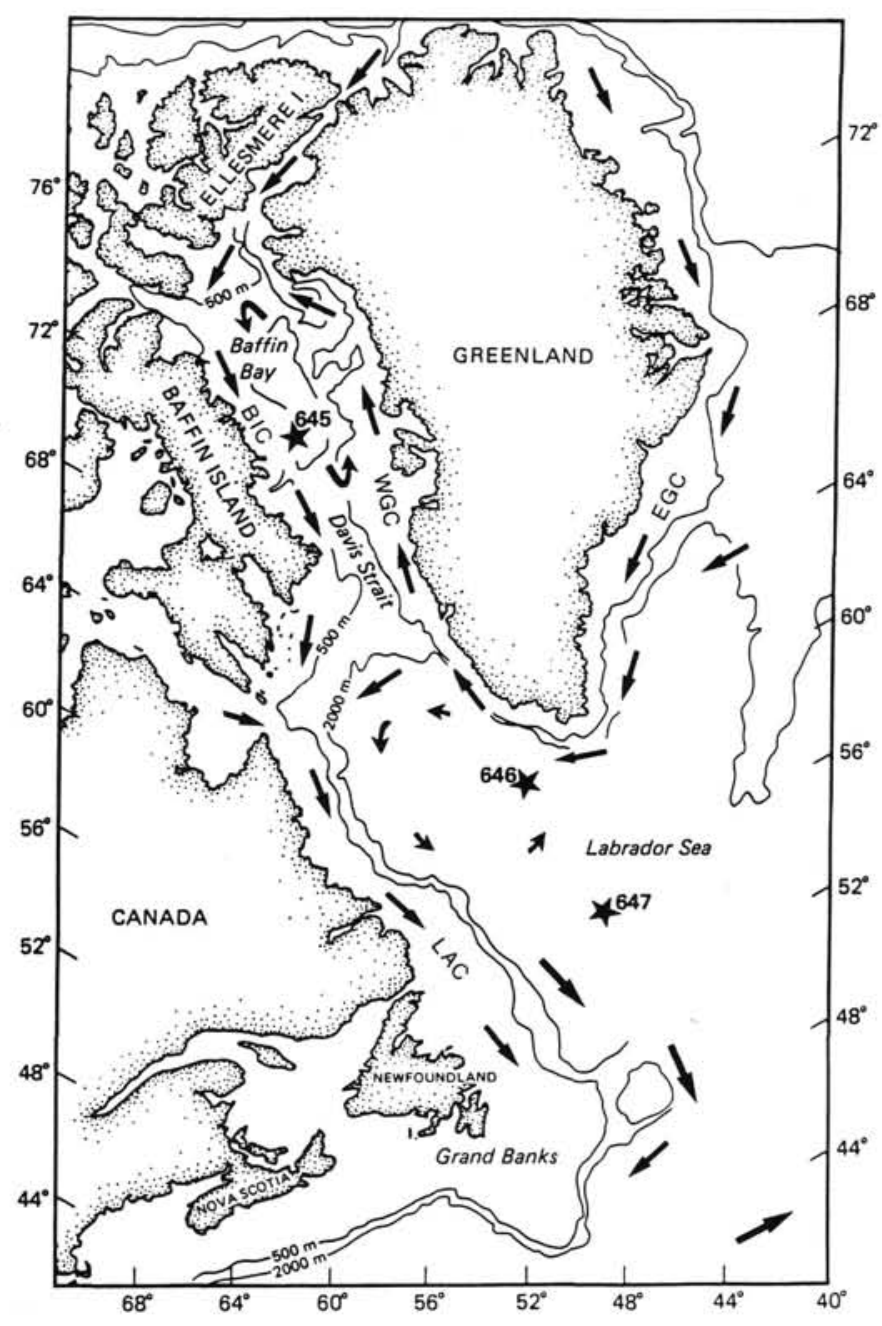

Figure 1. Location map of Sites 645,646 , and 647. Black arrows indicate surface-water circulation (according to Tschernia, 1982). BIC = Baffin Island Current; $L A C=$ Labrador Current; $E G C=$ East Greenland Current; $W G C=$ West Greenland Current.

North Atlantic Ocean (the West Greenland Current) (Fig. 1; Tchernia, 1982; Williams, 1986). This circulation is counterclockwise (cyclonic) (Fig. 1; Marko et al., 1982; Williams, 1986). Between December and April, Baffin Bay is covered by sea ice, whereas during the rest of the year, drifting icebergs are of major importance (Marko et al., 1982; Williams, 1986; observations, Leg 105).

Surface-water circulation in the Labrador Sea is dominated by the Greenland Current System (i.e., the East and West Greenland currents) and the Labrador Current. The Labrador Sea is a zone of active mixing between polar water and water of tropical origin. Sea ice and icebergs occur occasionally in winter and spring (Tchernia, 1982).

\section{METHODS}

\section{Organic and Inorganic Carbon}

The diagrams of total organic carbon (TOC) combine data sets that were produced at three different laboratories (Tables 1-5).

The following procedure was used aboard the JOIDES Resolution. After dissolution of inorganic carbon by hydrochloric acid treatment, the carbonate-free residues were washed and dried. Then, the organiccarbon content of these carbonate-free residues was determined by means of a Perkin Elmer 240C Elemental Analyzer. The organic-carbon values obtained by this procedure (i.e., $\mathrm{TOC}^{\prime}$ in wt $\%$ of the carbonate-free sediment fraction) were transposed into TOC values ( $w t \%$ of the bulksediment sample), using the equation,

$$
T O C=\frac{100-(8.334 \times T C)}{\left(100 / T O C^{\prime}\right)-8.334},
$$

where $T C$ is the total carbon in wt $\%$ of the bulk sediment, as determined by the Elemental Analyzer.

The same procedure was used at the IGL Giessen laboratory; however, the analyses were performed using a Heraeus CHN Analyzer. At the Jülich Institute, TOC was determined using a LECO IR-112 Carbon Analyzer after treatments with hydrochloric acid to remove the inorganic carbon.

To test whether all three data sets could be combined in one diagram, some samples were analyzed by different methods. The results indicated that the TOC values obtained in the different laboratories agreed well (Fig. 3).

The inorganic carbon (IC) was calculated by difference:

$$
I C(\%)=T C(\%)-T O C(\%) .
$$

Then, the carbonate content was calculated as

$$
\mathrm{CaCO}_{3}(\%)=I C(\%) \times 8.334 .
$$

\section{Rock-Eval Pyrolysis}

Rock-Eval pyrolysis was performed according to the method described by Espitalié et al. (1977). The hydrogen and oxygen indices (HI and OI) were plotted in a "van Krevelen-type" diagram (Tissot and Welte, 1984). The HI vs. depth diagram combines data from shipboard and shore-based analyses. The good correlation between shipboard and shore-based data obtained from the same samples justifies the combination of both data sets into one plot (Fig. 4).

Several Rock-Eval measurements were performed (for method of kerogen concentration, see below). HI values of these kerogen concentrates are generally higher (see HI* in Fig. 5), but show the same $\mathrm{T}_{\max }$ values (Table 3 ) and the same trends as the data obtained from the bulk sediment (Fig. 5, Table 3). Kerogen concentrates from sediments with low $\mathrm{HI}^{*}$ values display higher, but also relatively low, $\mathrm{HI}^{*}$ values.

\section{Kerogen Microscopy}

A microscopic study of the kerogen fraction was performed only on samples from Site 645 because of too low organic-carbon contents at Sites 646 and 647 (see next).

The kerogen was concentrated by treating the samples successively with $\mathrm{HCl}$ and $\mathrm{HF}$ to remove carbonates and silicates, respectively (Durand, 1980). Afterwards, the carbonate- and silicate-free samples were washed with distilled water to neutrality. The dried material was embedded in a resin, and the resulting block was polished using successively finer abrasives. Maceral analyses were performed on these blocks by a two-scanning method in reflected light and in fluorescent light (see Littke, 1987). The macerals were classified according to the nomenclature described by Stach et al. (1982) and Hutton et al. (1980). Because of the large number of samples in this study, only about 100 macerals were counted in each sample. Therefore, the maceral percentages should only be considered rough estimates of the quality of the organic matter.

To assess maturity, vitrinite reflectivities were measured using particles larger than $10 \mu \mathrm{m}(\lambda=546 \mathrm{~nm}$, oil immersion; see Stach et al., 1982, p. 365).

\section{Estimates of Surface-Water Productivity}

Müller and Suess (1979) published a formula for estimating paleoproductivity values based on the relationship between organic-carbon accumulation rates of surface sediments, bulk-sedimentation rates, and measured (i.e., recent) surface-water productivity values. From more recent studies of sediment-trap data, Suess (1980) and Betzer et al. (1984) showed that only a small amount of the organic matter produced in surface waters reaches the seafloor. This means that only this amount is the maximum available for burial in the sediment. Combining the results of Müller and Suess (1979) and Betzer et al. (1984), the following formula was obtained: 

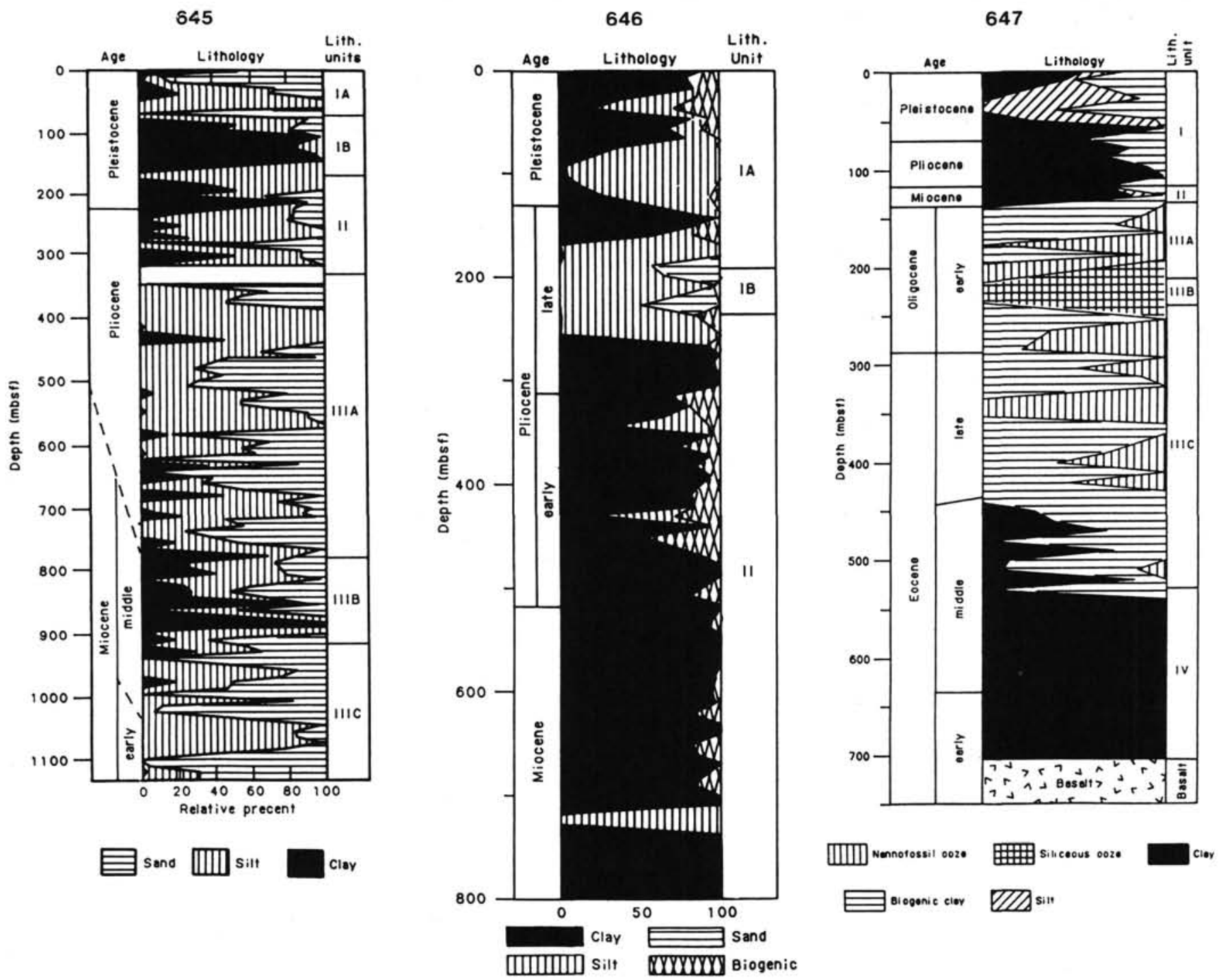

Figure 2. Summary diagrams of lithologic units at Sites 645, 646, and 647 (Srivastava, Arthur, et al., 1987).

$$
\begin{aligned}
P R \equiv & 5.31 \times\left[C \times\left(W B D-1.026 \times P O \times 10^{-2}\right)\right]^{0.71} \\
& \times S R^{0.07} \times D E P^{0.45},
\end{aligned}
$$

where $P R=$ paleoproductivity in $\mathrm{gC} \cdot \mathrm{m}^{-2} \cdot \mathrm{y}^{-1}, C=$ (marine) organic carbon in $\mathrm{wt} \%, W B D=$ wet-bulk density in $\mathrm{g} \cdot \mathrm{cm}^{-3}, S R=$ sedimentation rate in $\mathrm{cm}(\mathrm{k} . \mathrm{y} .)^{-1}, P O=$ porosity in \%, and $D E P=$ paleowater depth of the seafloor in meters. The detailed deduction of this formula is published in Stein (1986a). When using the paleoproductivity formula, the following points must be considered:

1. The equation is meaningful only if the sediments were deposited in oxic deep-water environments.

2. The content of marine organic matter must be known. We estimated this parameter from kerogen microscopy data (see below).

3. The sediments must have been deposited below the euphotic zone (see Betzer et al., 1984).

4. Diagenetic losses of organic matter (approximately up to $30 \%$; Raiswell and Berner, 1987) have not been considered in the formula until now, which means that past estimates of paleoproductivity may be somewhat low. Furthermore, note that in Equation 4 the term $S R$ has an exponent as low as 0.07 . This implies that an increase of the sedimentation rate by a factor of 100 will result in an increase of calculated pro- ductivity by a factor of only 1.38 . This is important to note because the sedimentation rate is a parameter that could not be determined accurately, especially at Site 645 (Srivastava, Arthur, et al., 1987).

\section{RESULTS}

\section{Quantity of Organic Carbon}

Almost all of the sediment sequence at Site 645 is characterized by TOC values that are distinctly higher than those recorded in open-marine environments. Most of the TOC values at Site 645 are higher than $0.5 \%$ and range between $0.5 \%$ and almost 3\% (Fig. 6, Tables 1 through 3). The open-ocean Sites 646 and 647 are characterized by predominantly low TOC values that range between less than 0.1 and $0.75 \%$ (Figs. 7 and 8 , Tables 4 and 5).

At Site 645, the lower TOC values are concentrated in the upper $320 \mathrm{~m}$, in the late Pliocene to Holocene sediments (Fig. 6). Between 780 and 340 mbsf (i.e., in the late Miocene to early Pliocene time interval), most TOC values vary between $0.5 \%$ and $1.5 \%$ (with a mean value of about $1 \%$ ). Maximum TOC values 
Table 1. Summary of total carbon, total organic carbon, carbonate content, and organic-carbon/total-nitrogen ratios in sediments from Site 645 .

\begin{tabular}{|c|c|c|c|c|c|c|}
\hline $\begin{array}{l}\text { Core/section } \\
\text { interval }(\mathrm{cm})\end{array}$ & $\begin{array}{l}\text { Depth } \\
\text { (mbsf) }\end{array}$ & $\mathrm{C}_{\text {tot }}$ & $\mathrm{C}_{\text {org }}{ }^{\prime}$ & $\mathrm{C}_{\text {org }}$ & $\mathrm{CaCO}_{3}$ & $\mathrm{C} / \mathrm{N}$ \\
\hline $105-645 B-3 X-2,106$ & 16.06 & 6.18 & 0.80 & 0.42 & 48.0 & 20 \\
\hline $6 \mathrm{X}-1,30$ & 42.90 & 6.61 & 0.33 & 0.15 & 53.8 & 6 \\
\hline $11 X-2,109$ & 93.49 & 2.30 & 0.22 & 0.18 & 17.7 & 7 \\
\hline $13 X-4,73$ & 115.52 & 7.06 & 0.97 & 0.43 & 55.2 & 21 \\
\hline $14 X-2,14$ & 121.54 & 3.09 & & & & \\
\hline $14 X-2,89$ & 122.29 & 5.74 & 0.42 & 0.23 & 45.9 & 8 \\
\hline $17 \mathrm{X}, \mathrm{CC}(17)$ & 158.29 & 4.65 & 0.45 & 0.29 & 36.4 & 11 \\
\hline $18 X-2,33$ & 160.23 & 4.12 & 1.12 & 0.81 & 27.6 & 16 \\
\hline $19 X-2,65$ & 170.25 & 2.27 & 0.55 & 0.47 & 15.0 & 7 \\
\hline 19X-6, 77 & 176.37 & 1.65 & 0.38 & 0.34 & 10.9 & 8 \\
\hline $19 X-6,91$ & 176.51 & 3.00 & 0.38 & 0.29 & 22.5 & 6 \\
\hline $22 X-2,43$ & 198.73 & 2.80 & 1.20 & 1.02 & 14.8 & 13 \\
\hline $28 X-2,145$ & 257.85 & 1.63 & 0.82 & 0.76 & 7.2 & 14 \\
\hline $29 X-1,93$ & 265.53 & 4.99 & 1.11 & 0.71 & 35.6 & 11 \\
\hline 105-645E-2R-1, 25 & 311.05 & 3.17 & 0.99 & 0.79 & 19.8 & 11 \\
\hline $4 \mathrm{R}-2,128$ & 458.48 & 1.32 & 0.99 & 0.96 & 3.0 & 17 \\
\hline $6 \mathrm{R}-2,30$ & 476.30 & 0.85 & 0.74 & 0.73 & 1.0 & 11 \\
\hline $8 \mathrm{R}-2,36$ & 495.56 & 0.87 & 0.81 & 0.81 & 0.5 & 12 \\
\hline $10 \mathrm{R}-3,97$ & 516.97 & 1.11 & 1.11 & 1.11 & 0.0 & 8 \\
\hline $12 \mathrm{R}-1,113$ & 533.33 & 1.16 & 1.14 & 1.14 & 0.2 & 10 \\
\hline $13 \mathrm{R}-3,98$ & 545.78 & 4.88 & 1.05 & 0.68 & 35.0 & 11 \\
\hline $13 R-3,115$ & 545.95 & 1.48 & 0.74 & 0.69 & 6.6 & 9 \\
\hline $14 \mathrm{R}-5,98$ & 558.48 & 0.98 & 0.98 & 0.98 & 0.0 & 12 \\
\hline $14 \mathrm{R}-6,14$ & 559.14 & & 0.44 & 0.44 & & 7 \\
\hline $16 \mathrm{R}-2,23$ & 572.53 & 1.09 & 1.09 & 1.09 & 0.0 & 16 \\
\hline $17 \mathrm{R}-2,08$ & 582.08 & 5.12 & 1.09 & 0.69 & 36.9 & 9 \\
\hline 17R-2, 27 & 582.27 & 0.99 & 0.76 & 0.74 & 2.0 & 11 \\
\hline $18 \mathrm{R}-2,25$ & 591.85 & 0.90 & 0.90 & 0.90 & 0.0 & 11 \\
\hline $18 \mathrm{R}-2,111$ & 592.71 & & 0.61 & 0.61 & & 15 \\
\hline $19 \mathrm{R}-2,127$ & 602.57 & 2.13 & 2.13 & 2.13 & 0.0 & 15 \\
\hline $19 \mathrm{R}-3,84$ & 603.64 & 1.60 & 0.76 & 0.70 & 7.5 & 11 \\
\hline $20 \mathrm{R}-2,22$ & 611.02 & 0.44 & 0.42 & 0.42 & 0.2 & 21 \\
\hline $21 \mathrm{R}-2,133$ & 621.73 & & 0.79 & 0.79 & & 20 \\
\hline $22 \mathrm{R}-2,33$ & 626.23 & 0.96 & 0.86 & 0.85 & 0.9 & 12 \\
\hline 23R-2, 64 & 630.52 & 1.00 & 0.84 & 0.83 & 1.4 & 17 \\
\hline $25 \mathrm{R}-2,51$ & 649.71 & 1.67 & 0.77 & 0.71 & 8.0 & 13 \\
\hline $27 R-2,100$ & 669.20 & 0.78 & 0.74 & 0.74 & 0.4 & 12 \\
\hline $29 \mathrm{R}-2,59$ & 688.09 & 1.05 & 0.93 & 0.92 & 1.1 & 19 \\
\hline $31 \mathrm{R}-5,140$ & 707.85 & & 0.41 & 0.41 & & 8 \\
\hline $33 \mathrm{R}-3,64$ & 718.34 & 2.11 & 1.34 & 1.24 & 7.2 & 10 \\
\hline $33 \mathrm{R}-3,82$ & 718.52 & 0.90 & 0.78 & 0.77 & 1.1 & 39 \\
\hline $34 \mathrm{R}-1,99$ & 725.39 & 0.82 & 0.82 & 0.82 & 0.0 & 16 \\
\hline $35 \mathrm{R}-2,137$ & 736.87 & 1.17 & 1.17 & 1.17 & 0.0 & 29 \\
\hline $37 \mathrm{R}-2,98$ & 755.88 & 2.00 & 1.06 & 0.97 & 8.6 & 11 \\
\hline $38 \mathrm{R}-6,30$ & 770.80 & 0.96 & 0.78 & 0.77 & 1.6 & 9 \\
\hline $39 \mathrm{R}-2,60$ & 774.40 & 1.60 & 0.78 & 0.72 & 7.3 & 10 \\
\hline $17 \mathrm{R}-3,125$ & 784.75 & 1.39 & 1.33 & 1.32 & 0.6 & 11 \\
\hline $40 \mathrm{R}-2,116$ & 784.96 & 1.14 & 1.14 & 1.14 & 0.0 & 29 \\
\hline $41 \mathrm{R}-3,77$ & 795.67 & 1.23 & 1.23 & 1.23 & 0.0 & 31 \\
\hline $42 \mathrm{R}-4,84$ & 806.94 & 1.12 & 0.53 & 0.50 & 5.1 & 27 \\
\hline $43 R-5,75$ & 817.95 & 1.16 & 1.16 & 1.16 & 0.0 & 29 \\
\hline $44 \mathrm{R}-6,60$ & 828.90 & 1.57 & 1.51 & 1.50 & 0.6 & 17 \\
\hline $45 R-5,50$ & 837.00 & 1.25 & 1.12 & 1.11 & 1.2 & 28 \\
\hline $46 \mathrm{R}-4,79$ & 845.39 & 1.76 & 1.34 & 1.29 & 3.9 & 17 \\
\hline $48 R-3,76$ & 863.16 & 0.99 & 0.79 & 0.78 & 1.8 & 10 \\
\hline $49 \mathrm{R}-2,85$ & 870.95 & 1.58 & 1.41 & 1.39 & 1.6 & 18 \\
\hline $50 \mathrm{R}-2,128$ & 880.88 & 1.63 & 1.45 & 1.43 & 1.7 & 11 \\
\hline $51 \mathrm{R}-1,66$ & 885.76 & 1.99 & 1.75 & 1.71 & 2.3 & 22 \\
\hline $52 \mathrm{R}-4,6$ & 892.36 & 1.53 & 1.47 & 1.46 & 0.6 & 13 \\
\hline $53 \mathrm{R}-4,102$ & 903.12 & 1.60 & 1.60 & 1.60 & 0.0 & 20 \\
\hline $54 \mathrm{R}-3,92$ & 911.12 & 1.87 & 1.78 & 1.76 & 0.9 & 16 \\
\hline $55 \mathrm{R}-3,106$ & 920.86 & 3.06 & 2.59 & 2.46 & 5.0 & 16 \\
\hline $56 \mathrm{R}-5,57$ & 933.18 & 1.84 & 1.60 & 1.56 & 2.3 & 16 \\
\hline $57 \mathrm{R}-2,113$ & 938.83 & 1.09 & 1.09 & 1.09 & 0.0 & 12 \\
\hline $58 \mathrm{R}-1,27$ & 946.17 & 1.63 & 1.48 & 1.46 & 1.4 & 13 \\
\hline $58 \mathrm{R}-3,126$ & 951.16 & 1.23 & 1.23 & 1.23 & 0.0 & 11 \\
\hline $59 \mathrm{R}-3,124$ & 959.84 & 3.58 & & & & \\
\hline $60 \mathrm{R}-4,138$ & 971.18 & 1.29 & 1.29 & 1.29 & 0.0 & 14 \\
\hline $61 \mathrm{R}-3,41$ & 978.31 & & 0.82 & 0.82 & & 27 \\
\hline $61 \mathrm{R}-6,24$ & 982.64 & 0.83 & 0.83 & 0.83 & 0.0 & 21 \\
\hline $62 R-5,33$ & 989.83 & 1.37 & 1.10 & 1.07 & 2.5 & 14 \\
\hline $63 R-3,41$ & 997.61 & 0.80 & 0.76 & 0.76 & 0.4 & 19 \\
\hline $64 \mathrm{R}-1,13$ & 1004.03 & 1.11 & 1.11 & 1.11 & 0.0 & 28 \\
\hline $65 \mathrm{R}-2,117$ & 1016.27 & 1.47 & 1.47 & 1.47 & 0.0 & 12 \\
\hline $66 \mathrm{R}-1,111$ & 1024.41 & 1.38 & 1.38 & 1.38 & 0.0 & 13 \\
\hline
\end{tabular}

Table 1 (continued).

\begin{tabular}{ccccccr}
\hline $\begin{array}{c}\text { Core/section } \\
\text { interval }(\mathrm{cm})\end{array}$ & $\begin{array}{c}\text { Depth } \\
\text { (mbsf) }\end{array}$ & $\mathrm{C}_{\text {tot }}$ & $\mathrm{C}_{\text {org }}$ & $\mathrm{C}_{\text {org }}$ & $\mathrm{CaCO}_{3}$ & $\mathrm{C} / \mathrm{N}$ \\
\hline 67R-3, 41 & 1036.31 & 1.64 & 1.64 & 1.64 & 0.0 & 13 \\
68R-2, 123 & 1045.33 & 2.02 & 1.98 & 1.97 & 0.4 & 17 \\
69R-3, 30 & 1054.50 & 1.23 & 1.23 & 1.23 & 0.0 & 21 \\
69R-3, 124 & 1056.44 & & 1.68 & 1.68 & & 19 \\
$70 \mathrm{R}-2,125$ & 1064.05 & 1.28 & 1.28 & 1.28 & 0.0 & 14 \\
$71 \mathrm{R}-2,133$ & 1075.93 & 0.80 & 0.80 & 0.80 & 0.0 & 20 \\
$72 \mathrm{R}-2,51$ & 1082.71 & 0.70 & 0.70 & 0.70 & 0.0 & 18 \\
$73 \mathrm{R}-4,73$ & 1095.63 & 0.86 & 0.86 & 0.86 & 0.0 & 22 \\
$74 \mathrm{R}-2,136$ & 1102.66 & 1.23 & 1.23 & 1.23 & 0.0 & 15 \\
$75 \mathrm{R}-2,114$ & 1111.91 & 0.57 & & & & \\
$76 \mathrm{R}-2,41$ & 1120.71 & 0.27 & 0.27 & 0.27 & 0.0 & 5 \\
$76 \mathrm{R}-4,106$ & 1124.36 & 0.97 & 0.97 & 0.97 & 0.0 & 11 \\
$77 \mathrm{R}-2,17$ & 1129.77 & 1.08 & 1.08 & 1.08 & 0.0 & 8 \\
$78 \mathrm{R}-3,114$ & 1141.84 & 0.76 & 0.76 & 0.76 & 0.0 & 13 \\
\hline
\end{tabular}

$\mathrm{C}_{\mathrm{tot}}=$ total carbon; $\mathrm{C}_{\mathrm{org}^{\prime}}=$ total organic carbon as a percentage of carbonate-free sample; $\mathrm{C}_{\mathrm{org}}=$ total organic carbon as percentage of whole sample: $\mathrm{CaCO}_{3}=$ carbonate content; $\mathrm{C} / \mathrm{N}=$ ratio of organic carbon/total nitrogen. $\mathrm{C}_{\text {org }}$, also equals TOC' $^{\prime}$ in Equation 1.

occur between a depth of 1040 and 780 mbsf in middle Miocene sediments that range between $1 \%$ and $3 \%$ (Fig. 6). In the lowermost $100 \mathrm{~m}$ of the sequence, the TOC content decreases, and values vary between $0.5 \%$ and $1.2 \%$ (Fig. 6).

At Site 646, the upper Miocene to lower Pliocene section is characterized by low-amplitude TOC variations that range between about $0.25 \%$ and $0.45 \%$, with lower values in the lower part of the section. The following upper Pliocene to Holocene interval shows high-amplitude variations between $0.15 \%$ and $0.75 \%$ (Fig. 7).

At Site 647 , low TOC values ranging between $0.05 \%$ and $0.3 \%$ are recorded for the Eocene. Some higher organic-carbon contents $(0.3 \%$ to $0.55 \%)$ are concentrated in the early Oligocene and the late Pliocene. In the Miocene and Pleistocene time intervals, the TOC values again are dominated by low values of less than $0.3 \%$ (Fig. 8).

In the right half of Figures 6 and 7, short-term fluctuations in organic-carbon content with periods of 20,000 to $100,000 \mathrm{yr}$ are shown for several time intervals at Sites 645 and 646, respectively. These cycles are also shown in the carbonate records of Sites 645 and 646 (Tables 2 and 4).

Because changes in the percentage values of TOC can result from changes in the abundance of both mineral components and organic-carbon content, the percentage values were transposed into accumulation rates (Srivastava, Arthur, et al., 1987; for method see van Andel et al., 1975). Using these accumulation rates, which are expressed in units of mass per area and time (i.e., $\mathrm{gC} \cdot \mathrm{cm}^{-2} \mathrm{k} . \mathrm{y} .{ }^{-1}$ ), dilution effects from nonorganiccarbon components can be excluded. Note that to calculate accumulation rates, mean sedimentation rates for the specific time intervals must be used. Thus, the accumulation rates of organic carbon shown in Figure 9 should also be used as mean accumulation rates, and single spikes should not be overinterpreted.

At Site 645 , the accumulation rates of TOC vary between 0.03 and $0.3 \mathrm{gC} \cdot \mathrm{cm}^{-2} \cdot \mathrm{k} . \mathrm{y} .^{-1}$ (Fig. 8). Two maxima of organic-carbon accumulation are recorded in the middle Miocene (between 18 and $12.5 \mathrm{Ma}$ ) and the late Pliocene to Holocene (3.4 and $0 \mathrm{Ma}$ ). The distinct increase at about $3.4 \mathrm{Ma}$ is also observed in the inorganic sediment fraction, i.e., in the (mainly terrigenous) carbonate and siliciclastic components (Srivastava, Arthur, et al., 1987, p.149). The middle Miocene maximum is based on a minimum mean sedimentation rate of $42 \mathrm{~m} / \mathrm{m}$.y. Because of the limited age constraints for the interval between 750 mbsf and the bottom of Hole 645 (Srivastava, Arthur, et al. 1987 , p. 103), the exact numbers of accumulation rates and ages 
shown in Figure 9 should be used cautiously. However, a middle Miocene maximum for organic-carbon accumulation is definite. If the estimated middle Miocene sedimentation rate is too low, than the estimated accumulation rates are also too low. This means that the real TOC accumulation rates for the middle Miocene were even higher; thus, the middle Miocene maximum should become more distinct.

At Sites 646 and 647 , the accumulation rates of organic carbon are distinctly lower than those recorded at Site 645 (Fig. 9). Very low accumulation rates of less than $0.04 \mathrm{gC} \cdot \mathrm{cm}^{-2}$. k.y. ${ }^{-1}$ characterize the time intervals between 9 and $7 \mathrm{Ma}$ and between $3.2 \mathrm{Ma}$ and the Holocene at Site 646. At this site, the highest accumulation rates between 0.04 and $0.07 \mathrm{gC} \cdot \mathrm{cm}^{-2}$. k.y. ${ }^{-1}$ occur between 7 and 3.2 Ma (Fig. 9).

At Site 647, the Tertiary record is incomplete because of three major hiatuses (Fig. 9). Organic-carbon accumulation rates are lower than $0.03 \mathrm{gC} \cdot \mathrm{cm}^{-2} \cdot \mathrm{k} . \mathrm{y}^{-1}$ throughout the entire time intervals documented in the sediment record. Minimum values occur between 55 and $45 \mathrm{Ma}, 35$ and $30 \mathrm{Ma}$, and 20 and $15 \mathrm{Ma}$ (less than $0.003 \mathrm{gC} \cdot \mathrm{cm}^{-2} \cdot$ k.y. ${ }^{-1}$; Fig. 9). Between 45 and 35 $\mathrm{Ma}$ and during the last $3 \mathrm{Ma}$, accumulation rates vary between 0.01 and $0.03 \mathrm{gC} \cdot \mathrm{cm}^{-2} \cdot$ k.y. ${ }^{-1}$ (Fig. 9).

\section{Quality of Organic Matter}

To reconstruct the history of organic-carbon deposition, one must know not only the quantity, but also the quality of the organic matter. This means that the amount of marine and terrigenous organic matter must be determined. Here, we used $\mathrm{C} / \mathrm{N}$ ratios, Rock-Eval pyrolysis, and kerogen microscopy to obtain information about the type of organic matter in the sediments (for gas-chromatography (GC) and gas-chromatography/massspectrometry (GC/MS) data see Ten Haven and Rullkötter, this volume).

\section{Organic Carbon/Nitrogen Ratios}

Organic carbon to total nitrogen $(\mathrm{C} / \mathrm{N})$ ratios are frequently used to characterize the type of organic matter found in deepsea sediments. This is possible because different groups of organisms produce organic matter that contain different carbon and nitrogen contents. The mean $\mathrm{C} / \mathrm{N}$ ratios for marine zooplankton and marine phytoplankton is about 6 , whereas higher land plants have $\mathrm{C} / \mathrm{N}$ ratios of more than 15 (e.g., Bordowskiy, 1965a, 1965b; Scheffer and Schachtschabel, 1984). However, one must consider that the $\mathrm{C} / \mathrm{N}$ ratios may change during diagenesis (see Müller, 1977; Emery and Uchupi, 1984). Furthermore, in organic-carbon-poor sediments, the amount of inorganic nitrogen (fixed as ammonium ions in the interlayers of clay minerals) may result in $\mathrm{C} / \mathrm{N}$ ratios that are too low (Müller, 1977). Thus, $\mathrm{C} / \mathrm{N}$ ratios must be used cautiously if these are the only parameters available to characterize the quality of the organic matter.

The sediments of Site 645 are characterized by generally high $\mathrm{C} / \mathrm{N}$ ratios of more than 10 . Most of the values vary between 10 and 30 (Fig. 10, Table 1), indicating the predominance of terrigenous organic matter at Site 645 . However, values around 10 may imply some admixtures of marine compounds.

At Sites 646 and 647 , the $\mathrm{C} / \mathrm{N}$ ratios are distinctly lower than those recorded at Site 645 . Most of these ratios vary between 10 and 4 (Fig. 10; Tables 4 and 5). This may suggest the predominance of marine organic matter at both sites. However, because of very low organic-carbon and nitrogen values recorded in most samples, inorganic-nitrogen contents may have influenced the $\mathrm{C} / \mathrm{N}$ ratios significantly (see Müller, 1977). This means that until inorganic-nitrogen values are available, the $\mathrm{C} / \mathrm{N}$ ratios of the organic-carbon-poor sediments at Sites 646 and 647 should be interpreted cautiously.

\section{Rock-Eval Pyrolysis}

The results of Rock-Eval pyrolysis are plotted in a van Krevelen-type diagram (Fig. 11; Tissot and Welte, 1984). These data indicate that most of the organic matter in sediments from Holes $645 \mathrm{~B}$ and $645 \mathrm{D}$ (i.e., in sediments of the upper $460 \mathrm{~m}$ ) is of hydrogen-poor type III, i.e., of terrigenous origin. At Hole $645 \mathrm{E}$, i.e., below a depth of $460 \mathrm{mbsf}$, we found higher HI values, which indicates an admixture of dominant terrestrial and minor marine organic matter (Figs. 11 and 12, Table 3).

Because of the low organic-carbon content of Site 647 samples, only a few Rock-Eval analyses were performed on these sediments. The HI values of these sediments are very low, ranging between 43 and 92 (Table 6). These low HI values and the high OI values suggest a type of organic matter that is highly oxidized (T. Cederberg, pers. comm., 1987). The Rock-Eval data derived from Site 646 samples is very similar to that of Site 647 (T. Cederberg, pers. comm., 1987).

\section{Kerogen Microscopy}

Results from kerogen microscopy are summarized in Table 3. In general, the data corroborate the Rock-Eval and $\mathrm{C} / \mathrm{N}$ results. Terrigenous macerals dominate the organic matter at Site 645 . Values vary between $50 \%$ and almost $100 \%$ of the total macerals (Table 3). Among these macerals, vitrinites (huminites) and sporinites are most abundant, whereas brightly reflecting inertinites and cutinites or resinites are rare. Minor amounts of cutinites occur in samples between 900 and 1000 mbsf. Inertinite is most abundant at depths between about 400 and $550 \mathrm{mbsf}$, which indicates a stronger degradation (oxidation) of the terrestrial organic matter. This is supported by most vitrinites in sediments from 0 to $500 \mathrm{mbsf}$, which display a bright reflectance that corresponds to an evolutionary stage between that of "true" vitrinite and that of "true" inertinite. Such particles are often interpreted as reworked organic matter derived from the erosion and redeposition of kerogen from older sedimentary rocks or from more mature coals. In our samples, however, brightly reflecting particles as well as darker vitrinites are rather large, i.e., often larger than $0.05 \mathrm{~mm}$ in diameter. This indicates similar age and transporting energies for both kinds of fragments. Reworked kerogen fragments from older sedimentary strata should be smaller than indigenous vitrinites because the former were transported twice. We concluded that these abundant, brightly reflecting, organic particles derive from moderate oxidation of plant fragments during transport, rather than from reworking of kerogen from older strata.

Between 500 and 970 netlike liptinites are common particles ("Alginite" in Fig. 12). Although these cannot be grouped together with any of the petrographically well-known algae groups (i.e., Tasmanales), these liptinites are interpreted as derivatives of autochthonous marine organisms because (1) they show a characteristic, finely structured morphology similar to lamellar alginite, as described by Hutton et al. (1980) and are a potential product of benthic cyanobacteria, (2) they emit a green-yellow fluorescence brighter than that of terrestrial liptinites, and (3) their abundance does not seem to be correlated with the abundance of terrestrial organic particles.

However, the occurrence of major proportions of marine organic particles is not supported by GC/MS results of saturated hydrocarbons of Ten Haven and Rullkötter (this volume), who describe unusual, probably higher-plant-derived triterpenoids, but no steroids typical for phytoplankton, and few hopanoids typical of bacteria from the alginite-rich samples.

\section{Maturity of Organic Matter}

The reflectance values of primary vitrinite from Site 645 sediments are very low and vary between 0.25 and 0.31 (Table 3 ). 
Table 2 (continued).

Table 2. High-resolution records of total organic carbon and carbonate data from six densely sampled intervals at Site 645 .

\begin{tabular}{|c|c|c|c|c|c|}
\hline $\begin{array}{l}\text { Core/section } \\
\text { interval }(\mathrm{cm})\end{array}$ & $\begin{array}{l}\text { Depth } \\
\text { (mbsf) }\end{array}$ & $\mathrm{C}_{\text {tot }}$ & $\mathrm{C}_{\text {org' }}$ & $C_{\text {org }}$ & $\mathrm{CaCO}_{3}$ \\
\hline \multicolumn{6}{|l|}{ A } \\
\hline $105-645 \mathrm{~B}-1 \mathrm{X}-1,21$ & 0.21 & 5.03 & 0.15 & 0.09 & 41.18 \\
\hline $105-645 \mathrm{~B}-1 \mathrm{X}-1,44$ & 0.44 & 5.57 & 0.12 & 0.06 & 45.88 \\
\hline $105-645 \mathrm{~B}-1 \mathrm{X}-1,60$ & 0.60 & 5.39 & 0.15 & 0.08 & 44.22 \\
\hline 105-645B-1X-1, 80 & 0.80 & 3.23 & 0.21 & 0.16 & 25.62 \\
\hline 105-645B-1X-1, 101 & 1.01 & 5.58 & 0.19 & 0.10 & 45.64 \\
\hline 105-645B-1X-1, 120 & 1.20 & 0.65 & 0.49 & 0.48 & 1.39 \\
\hline 105-645B-1X-1, 140 & 1.40 & 0.69 & 0.40 & 0.39 & 2.50 \\
\hline 105-645B-1X-2, 12 & 1.62 & 0.49 & 0.27 & 0.26 & 1.88 \\
\hline $105-645 \mathrm{~B}-1 \mathrm{X}-2,32$ & 1.82 & 1.03 & 0.32 & 0.30 & 6.08 \\
\hline 105-645B-1X-1, 52 & 2.02 & 1.56 & 0.37 & 0.33 & 10.23 \\
\hline 105-645B-1X-1, 72 & 2.22 & 2.11 & 0.17 & 0.14 & 16.40 \\
\hline 105-645B-1X-2, 93 & 2.43 & 1.53 & 0.11 & 0.10 & 11.94 \\
\hline 105-645B-1X-2, 113 & 2.63 & 2.32 & 0.14 & 0.11 & 18.38 \\
\hline $105-645 \mathrm{~B}-1 \mathrm{X}-2,131$ & 2.81 & 2.62 & 0.14 & 0.11 & 20.91 \\
\hline 105-645B-1X-3, 11 & 3.11 & 3.20 & 0.23 & 0.17 & 25.24 \\
\hline $105-645 B-1 X-3,31$ & 3.31 & 3.30 & 0.13 & 0.10 & 26.71 \\
\hline $105-645 \mathrm{~B}-1 \mathrm{X}-1,50$ & 3.50 & 3.16 & 0.27 & 0.20 & 24.64 \\
\hline $105-645 \mathrm{~B}-1 \mathrm{X}-3,70$ & 3.70 & 4.77 & 0.26 & 0.16 & 38.42 \\
\hline \multicolumn{6}{|l|}{ B } \\
\hline 105-645B-11X-1, 20 & 91.10 & 3.39 & 0.19 & 0.14 & 27.10 \\
\hline $105-645 \mathrm{~B}-11 \mathrm{X}-1,40$ & 91.30 & 1.21 & 0.25 & 0.23 & 8.17 \\
\hline 105-645B-11X-1, 59 & 91.49 & 2.07 & 0.27 & 0.23 & 15.35 \\
\hline 105-645B-11X-1, 80 & 91.70 & 5.99 & 0.12 & 0.06 & 49.41 \\
\hline 105-645B-11X-1, 98 & 91.88 & 4.59 & 0.18 & 0.11 & 37.31 \\
\hline 105-645B-11X-1, 120 & 92.10 & 4.56 & 0.60 & 0.39 & 34.74 \\
\hline 105-645B-11X-1, 140 & 92.30 & 6.21 & 0.32 & 0.16 & 50.43 \\
\hline $105-645 \mathrm{~B}-11 \mathrm{X}-2,10$ & 92.50 & 3.10 & 0.37 & 0.28 & 23.48 \\
\hline $105-645 \mathrm{~B}-11 \mathrm{X}-2,30$ & 92.70 & 1.12 & 0.19 & 0.18 & 7.88 \\
\hline 105-645B-11X-2, 50 & 92.90 & 2.78 & 0.20 & 0.16 & 21.87 \\
\hline $105-645 \mathrm{~B}-11 \mathrm{X}-2,70$ & 93.10 & 6.43 & 0.26 & 0.12 & 52.56 \\
\hline 105-645B-11X-2, 89 & 93.29 & 1.57 & 0.16 & 0.14 & 11.91 \\
\hline $105-645 B-11 X-2,100$ & 93.40 & 1.19 & 0.27 & 0.25 & 7.84 \\
\hline $105-645 B-11 X-2,120$ & 93.60 & 2.29 & 0.15 & 0.12 & 18.06 \\
\hline $105-645 B-11 X-2,140$ & 93.80 & 6.13 & 0.29 & 0.15 & 49.88 \\
\hline $105-645 \mathrm{~B}-11 \mathrm{X}-3,10$ & 94.00 & 4.14 & 0.39 & 0.26 & 32.30 \\
\hline 105-645B-11X-3, 30 & 94.20 & 6.26 & 0.43 & 0.21 & 50.39 \\
\hline $105-645 \mathrm{~B}-11 \mathrm{X}-3,50$ & 94.40 & 6.36 & 0.26 & 0.12 & 51.96 \\
\hline 105-645B-11X-3, 70 & 94.60 & 3.45 & 0.19 & 0.94 & 20.91 \\
\hline 105-645B-11X-3, 91 & 94.81 & 5.96 & 0.61 & 0.32 & 46.97 \\
\hline 105-645B-11X-3, 110 & 95.00 & 1.10 & 0.27 & 0.25 & 7.08 \\
\hline $105-645 \mathrm{~B}-11 \mathrm{X}-3,130$ & 95.20 & 1.14 & 0.24 & 0.22 & 7.65 \\
\hline $105-645 B-11 X-3,150$ & 95.40 & 4.82 & 0.35 & 0.22 & 38.37 \\
\hline \multicolumn{6}{|l|}{ C } \\
\hline $105-645 B-14 X-1,20$ & 120.10 & 4.75 & 0.53 & 0.33 & 36.79 \\
\hline $105-645 \mathrm{~B}-14 \mathrm{X}-1,40$ & 120.30 & 5.11 & 0.35 & 0.21 & 40.86 \\
\hline $105-645 \mathrm{~B}-14 \mathrm{X}-1,60$ & 120.60 & 1.99 & 0.35 & 0.30 & 14.08 \\
\hline 105-645B-14X-1, 80 & 120.70 & 4.23 & 0.32 & 0.21 & 33.48 \\
\hline $105-645 \mathrm{~B}-14 \mathrm{X}-1,100$ & 120.90 & 3.62 & 0.22 & 0.16 & 28.86 \\
\hline 105-645B-14X-1, 120 & 121.10 & 4.36 & 0.42 & 0.28 & 34.03 \\
\hline $105-645 \mathrm{~B}-14 \mathrm{X}-1,140$ & 121,30 & 5.02 & 0.47 & 0.28 & 39.47 \\
\hline 105-645B-14X-2, 10 & 121.50 & 5.10 & 0.39 & 0.23 & 40.57 \\
\hline $105-645 \mathrm{~B}-14 \mathrm{X}-2,30$ & 121.70 & 5.41 & 0.26 & 0.15 & 43.87 \\
\hline $105-645 \mathrm{~B}-14 \mathrm{X}-2,50$ & 121.90 & 5.36 & 0.42 & 0.24 & 42.66 \\
\hline 105-645B-14X-2, 71 & 122.11 & 5.55 & 0.41 & 0.23 & 44.35 \\
\hline 105-645B-14X-2, 87 & 122.27 & 7.59 & 0.28 & 0.11 & 62.38 \\
\hline $105-645 \mathrm{~B}-14 \mathrm{X}-2,100$ & 122.40 & 5.08 & 0.54 & 0.33 & 39.62 \\
\hline $105-645 \mathrm{~B}-14 \mathrm{X}-2,120$ & 122.60 & 4.91 & 0.43 & 0.26 & 38.72 \\
\hline $105-645 \mathrm{~B}-14 \mathrm{X}-2,141$ & 122.81 & 3.61 & 0.35 & 0.25 & 27.99 \\
\hline $105-645 \mathrm{~B}-14 \mathrm{X}-3,20$ & 123.10 & 4.08 & 0.26 & 0.18 & 32.54 \\
\hline $105-645 \mathrm{~B}-14 \mathrm{X}-3,41$ & 123.31 & 4.20 & 0.31 & 0.21 & 33.28 \\
\hline $105-645 \mathrm{~B}-14 \mathrm{X}-3,60$ & 123.50 & 1.49 & 0.17 & 0.15 & 11.16 \\
\hline $105-645 \mathrm{~B}-14 \mathrm{X}-3,89$ & 123.79 & 1.56 & 0.18 & 0.16 & 11.68 \\
\hline $105-645 B-14 X-3,110$ & 124.00 & 5.17 & 0.56 & 0.33 & 40.30 \\
\hline
\end{tabular}

\begin{tabular}{|c|c|c|c|c|c|}
\hline $\begin{array}{l}\text { Core/section } \\
\text { interval }(\mathrm{cm})\end{array}$ & $\begin{array}{l}\text { Depth } \\
\text { (mbsf) }\end{array}$ & $\mathrm{C}_{\mathrm{tot}}$ & $\mathrm{C}_{\text {org' }}$ & $\mathrm{C}_{\text {org }}$ & $\mathrm{CaCO}_{3}$ \\
\hline \multicolumn{6}{|l|}{ D } \\
\hline $105-645 \mathrm{~B}-31 \mathrm{X}-1,40$ & 284.20 & 0.20 & 0.11 & 0.11 & 0.76 \\
\hline $105-645 \mathrm{~B}-31 \mathrm{X}-1,60$ & 284.40 & 0.23 & 0.09 & 0.09 & 1.18 \\
\hline $105-645 \mathrm{~B}-31 \mathrm{X}-1,80$ & 284.60 & 0.71 & 0.10 & 0.09 & 5.13 \\
\hline $105-645 \mathrm{~B}-31 \mathrm{X}-1,100$ & 284.80 & 2.27 & 0.24 & 0.20 & 17.26 \\
\hline $105-645 \mathrm{~B}-31 \mathrm{X}-1,130$ & 285.10 & 1.96 & 0.30 & 0.26 & 14.19 \\
\hline 105-645B-31X-1, 149 & 285.29 & 1.93 & 0.25 & 0.21 & 14.30 \\
\hline $105-645 \mathrm{~B}-31 \mathrm{X}-2,20$ & 285.50 & 2.50 & 0.14 & 0.11 & 19.90 \\
\hline $105-645 \mathrm{~B}-31 \mathrm{X}-2,38$ & 285.68 & 3.10 & 0.22 & 0.17 & 24.45 \\
\hline $105-645 \mathrm{~B}-31 \mathrm{X}-2,60$ & 285.90 & 2.65 & 0.26 & 0.21 & 20.36 \\
\hline 105-645B-31X-2, 92 & 286.22 & 2.25 & 0.40 & 0.34 & 15.95 \\
\hline 105-645B-31X-2, 110 & 286.40 & 2.29 & 0.27 & 0.22 & 17.22 \\
\hline $105-645 \mathrm{~B}-31 \mathrm{X}-2,120$ & 286.50 & 2.28 & 0.34 & 0.28 & 16.64 \\
\hline 105-645B-31X-2, 140 & 286.70 & 2.25 & 0.43 & 0.36 & 15.73 \\
\hline $105-645 \mathrm{~B}-31 \mathrm{X}-3,10$ & 286.90 & 2.46 & 0.26 & 0.21 & 18.74 \\
\hline 105-645B-31X-3, 30 & 287.10 & 2.39 & 0.27 & 0.22 & 18.07 \\
\hline $105-645 \mathrm{~B}-31 \mathrm{X}-3,52$ & 287.32 & 1.69 & 0.15 & 0.13 & 13.00 \\
\hline 105-645B-31X-3, 70 & 287.50 & 1.60 & 0.09 & 0.08 & 12.68 \\
\hline $105-645 \mathrm{~B}-31 \mathrm{X}-3,100$ & 287.80 & 1.86 & 0.18 & 0.15 & 14.21 \\
\hline $105-645 \mathrm{~B}-31 \mathrm{X}-3,120$ & 288.00 & 2.35 & 0.28 & 0.23 & 17.66 \\
\hline $105-645 \mathrm{~B}-31 \mathrm{X}-3,140$ & 288.20 & 3.27 & 0.57 & 0.44 & 23.62 \\
\hline $105-645 \mathrm{~B}-31 \mathrm{X}-4,10$ & 288.40 & 1.15 & 0.22 & 0.20 & 7.90 \\
\hline $105-645 \mathrm{~B}-31 \mathrm{X}-4,30$ & 288.60 & 1.08 & 0.24 & 0.22 & 7.14 \\
\hline $105-645 \mathrm{~B}-31 \mathrm{X}-4,50$ & 288.80 & 0.71 & 0.25 & 0.24 & 3.92 \\
\hline $105-645 \mathrm{~B}-31 \mathrm{X}-4,70$ & 289.00 & 0.82 & 0.23 & 0.22 & 5.01 \\
\hline $105-645 \mathrm{~B}-31 \mathrm{X}-4,90$ & 289.20 & 2.98 & 0.33 & 0.26 & 22.71 \\
\hline $105-645 \mathrm{~B}-31 \mathrm{X}-4,110$ & 289.40 & 3.45 & 0.37 & 0.27 & 26.49 \\
\hline $105-645 \mathrm{~B}-31 \mathrm{X}-4,128$ & 289.58 & 2.62 & 0.39 & 0.32 & 19.21 \\
\hline 105-645B-31X-5 & 289.80 & 3.98 & 0.18 & 0.12 & 32.15 \\
\hline $105-645 \mathrm{~B}-31 \mathrm{X}-5,20$ & 290.00 & 3.70 & 0.20 & 0.14 & 29.66 \\
\hline $105-645 \mathrm{~B}-31 \mathrm{X}-5,39$ & 290.19 & 3.70 & 0.23 & 0.16 & 29.48 \\
\hline $105-645 \mathrm{~B}-31 \mathrm{X}-5,60$ & 290.40 & 1.29 & 0.32 & 0.29 & 8.31 \\
\hline 105-645B-31X-5, 80 & 290.60 & 1.14 & 0.23 & 0.21 & 7.73 \\
\hline $105-645 \mathrm{~B}-31 \mathrm{X}-5,100$ & 290.80 & 2.63 & & & \\
\hline $105-645 B-31 X-5,120$ & 291.00 & 2.67 & & & \\
\hline $105-645 \mathrm{~B}-31 \mathrm{X}-5,141$ & 291.21 & 2.88 & 0.28 & 0.22 & 22.19 \\
\hline \multicolumn{6}{|l|}{$\mathrm{F}$} \\
\hline $105-645 \mathrm{E}-13 \mathrm{X}-1,10$ & 541.90 & 1.19 & 0.89 & 0.87 & 2.70 \\
\hline $105-645 \mathrm{E}-13 \mathrm{X}-1,30$ & 542.10 & 0.84 & 0.84 & 0.84 & 0.00 \\
\hline 105-645E-13X-1, 54 & 542.34 & 6.07 & 0.99 & 0.53 & 46.14 \\
\hline $105-645 \mathrm{E}-13 \mathrm{X}-1,70$ & 542.50 & 3.22 & 0.87 & 0.69 & 21.12 \\
\hline $105-645 \mathrm{E}-13 \mathrm{X}-1,90$ & 542.70 & 1.74 & 0.77 & 0.70 & 8.64 \\
\hline $105-645 \mathrm{E}-13 \mathrm{X}-1,110$ & 542.90 & 0.91 & 0.83 & 0.82 & 0.72 \\
\hline $105-645 \mathrm{E}-13 \mathrm{X}-1,130$ & 543.10 & 0.96 & 0.86 & 0.85 & 0.90 \\
\hline $105-645 \mathrm{E}-13 \mathrm{X}-1,148$ & 543.28 & 1.08 & 1.08 & 1.08 & 0.00 \\
\hline 105-645E-13X-2, 20 & 543.50 & 1.13 & 0.97 & 0.96 & 1.45 \\
\hline $105-645 \mathrm{E}-13 \mathrm{X}-2,40$ & 543.70 & 1.07 & 0.98 & 0.97 & 0.82 \\
\hline 105-645E-13X-2, 60 & 543.90 & 0.96 & 0.89 & 0.88 & 0.63 \\
\hline $105-645 \mathrm{E}-13 \mathrm{X}-2,80$ & 544.10 & 0.94 & 0.89 & 0.89 & 0.45 \\
\hline $105-645 \mathrm{E}-13 \mathrm{X}-2,100$ & 544.30 & 1.07 & 0.93 & 0.92 & 1.26 \\
\hline $105-645 \mathrm{E}-13 \mathrm{X}-2,120$ & 544.50 & 1.71 & 1.50 & 1.47 & 2.00 \\
\hline $105-645 \mathrm{E}-13 \mathrm{X}-2,140$ & 544.70 & 1.15 & 1.09 & 1.08 & 0.55 \\
\hline $105-645 \mathrm{E}-13 \mathrm{X}-3,10$ & 544.90 & 1.10 & 0.92 & 0.91 & 1.62 \\
\hline $105-645 \mathrm{E}-13 \mathrm{X}-3,30$ & 545.10 & 1.01 & 1.00 & 1.00 & 0.09 \\
\hline $105-645 \mathrm{E}-13 \mathrm{X}-3,54$ & 545.34 & 1.81 & 0.74 & 0.67 & 9.50 \\
\hline $105-645 \mathrm{E}-13 \mathrm{X}-3,71$ & 545.51 & 0.93 & 0.70 & 0.69 & 2.04 \\
\hline $105-645 \mathrm{E}-13 \mathrm{X}-3,90$ & 545.70 & 1.15 & 0.67 & 0.64 & 4.24 \\
\hline $105-645 \mathrm{E}-13 \mathrm{X}-3,110$ & 545.90 & 4.71 & 1.13 & 0.76 & 32.94 \\
\hline $105-645 \mathrm{E}-13 \mathrm{X}-3,130$ & 546.10 & 1.21 & 0.81 & 0.78 & 3.57 \\
\hline $105-645 \mathrm{E}-13 \mathrm{X}-4$ & 546.30 & 0.98 & 0.74 & 0.72 & 2.13 \\
\hline $105-645 \mathrm{E}-13 \mathrm{X}-4,20$ & 546.50 & 1.10 & 0.75 & 0.73 & 3.11 \\
\hline $105-645 \mathrm{E}-13 \mathrm{X}-4,40$ & 546.70 & 1.13 & 0.92 & 0.90 & 1.90 \\
\hline $105-645 \mathrm{E}-13 \mathrm{X}-4,60$ & 546.90 & 1.57 & 0.94 & 0.89 & 5.70 \\
\hline $105-645 \mathrm{E}-13 \mathrm{X}-4,80$ & 547.10 & 1.15 & 0.86 & 0.84 & 2.60 \\
\hline 105-645E-13X-4, 100 & 547.30 & 1.17 & 1.03 & 1.02 & 1.28 \\
\hline $105-645 \mathrm{E}-13 \mathrm{X}-4,120$ & 547.50 & 1.23 & 0.94 & 0.92 & 2.62 \\
\hline $105-645 \mathrm{E}-13 \mathrm{X}-4,130$ & 547.60 & 1.19 & 1.06 & 1.05 & 1.19 \\
\hline $105-645 \mathrm{E}-13 \mathrm{X}-5,10$ & 547.90 & 1.20 & 0.96 & 0.94 & 2.17 \\
\hline $105-645 \mathrm{E}-13 \mathrm{X}-5,30$ & 548.10 & 1.45 & 1.17 & 1.14 & 2.59 \\
\hline $105-645 \mathrm{E}-13 \mathrm{X}-5,54$ & 548.34 & 1.76 & 1.75 & 1.75 & 0.10 \\
\hline $105-645 \mathrm{E}-13 \mathrm{X}-5,73$ & 548.53 & 1.56 & 1.56 & 1.56 & 0.00 \\
\hline
\end{tabular}


Table 2 (continued).

\begin{tabular}{|c|c|c|c|c|c|}
\hline $\begin{array}{l}\text { Core/section } \\
\text { interval }(\mathrm{cm})\end{array}$ & $\begin{array}{l}\text { Depth } \\
\text { (mbsf) }\end{array}$ & $C_{\text {tot }}$ & $\mathrm{C}_{\text {org' }}$ & $C_{\text {org }}$ & $\mathrm{CaCO}_{3}$ \\
\hline \multicolumn{6}{|l|}{$\mathrm{F}$} \\
\hline 105-645E-33X-1, 97 & 714.67 & 1.11 & 1.00 & 0.99 & 1.00 \\
\hline $105-645 \mathrm{E}-33 \mathrm{X}-1,27$ & 714.97 & 1.02 & 1.02 & 1.02 & 0.00 \\
\hline $105-645 \mathrm{E}-33 \mathrm{X}-1,40$ & 715.10 & 1.28 & 1.08 & 1.06 & 1.83 \\
\hline $105-645 \mathrm{E}-33 \mathrm{X}-1,60$ & 715.30 & 1.30 & 1.16 & 1.15 & 1.29 \\
\hline $105-645 \mathrm{E}-33 \mathrm{X}-1,79$ & 715.49 & 1.12 & 0.97 & 0.96 & 1.36 \\
\hline $105-645 \mathrm{E}-33 \mathrm{X}-1,120$ & 715.90 & 1.17 & 1.02 & 1.01 & 1.37 \\
\hline $105-645 \mathrm{E}-33 \mathrm{X}-1,140$ & 716.10 & 1.08 & 0.93 & 0.92 & 1.36 \\
\hline $105-645 \mathrm{E}-33 \mathrm{X}-2,19$ & 716.39 & 0.84 & 0.63 & 0.62 & 1.85 \\
\hline $105-645 \mathrm{E}-33 \mathrm{X}-2,38$ & 716.58 & 0.76 & 0.61 & 0.60 & 1.32 \\
\hline $105-645 \mathrm{E}-33 \mathrm{X}-2,60$ & 716.80 & 0.81 & 0.63 & 0.62 & 1.58 \\
\hline $105-645 \mathrm{E}-33 \mathrm{X}-2,19$ & 717.00 & 0.77 & 0.60 & 0.59 & 1.49 \\
\hline $105-645 \mathrm{E}-33 \mathrm{X}-2,100$ & 717.20 & 0.89 & 0.80 & 0.79 & 0.80 \\
\hline $105-645 \mathrm{E}-33 \mathrm{X}-2,120$ & 717.40 & 1.08 & 0.80 & 0.78 & 2.50 \\
\hline $105-645 \mathrm{E}-33 \mathrm{X}-2,140$ & 717.60 & 0.65 & 0.60 & 0.60 & 0.44 \\
\hline $105-645 \mathrm{E}-33 \mathrm{X}-3,10$ & 717.80 & 0.52 & 0.42 & 0.42 & 0.86 \\
\hline $105-645 \mathrm{E}-33 \mathrm{X}-3,30$ & 718.00 & 0.75 & 0.71 & 0.71 & 0.35 \\
\hline $105-645 \mathrm{E}-33 \mathrm{X}-3,50$ & 718.20 & 0.85 & 0.67 & 0.66 & 1.59 \\
\hline $105-645 \mathrm{E}-33 \mathrm{X}-3,70$ & 718.40 & 0.71 & 0.56 & 0.55 & 1.31 \\
\hline $105-645 \mathrm{E}-33 \mathrm{X}-3,110$ & 718.80 & 0.95 & 0.83 & 0.82 & 1.07 \\
\hline $105-645 \mathrm{E}-33 \mathrm{X}-3,134$ & 719.04 & 1.03 & 0.85 & 0.84 & 1.61 \\
\hline $105-645 \mathrm{E}-33 \mathrm{X}-4,10$ & 719.30 & 1.32 & 0.99 & 0.96 & 3.00 \\
\hline $105-645 \mathrm{E}-33 \mathrm{X}-4,30$ & 719.50 & 1.25 & 0.97 & 0.95 & 2.54 \\
\hline $105-645 \mathrm{E}-33 \mathrm{X}-3,90$ & 719.60 & 0.74 & 0.74 & 0.74 & 0.00 \\
\hline $105-645 \mathrm{E}-33 \mathrm{X}-4,50$ & 719.70 & 1.08 & 0.91 & 0.90 & 1.53 \\
\hline $105-645 \mathrm{E}-33 \mathrm{X}-4,70$ & 719.90 & 1.44 & 1.11 & 1.08 & 3.03 \\
\hline $105-645 \mathrm{E}-33 \mathrm{X}-4,90$ & 720.10 & 1.43 & 1.30 & 1.28 & 1.22 \\
\hline $105-645 \mathrm{E}-33 \mathrm{X}-4,110$ & 720.30 & 0.87 & 0.67 & 0.66 & 1.77 \\
\hline $105-645 \mathrm{E}-33 \mathrm{X}-4,130$ & 720.50 & 0.84 & 0.83 & 0.83 & 0.09 \\
\hline $105-645 \mathrm{E}-33 \mathrm{X}-4,148$ & 720.68 & 0.73 & 0.73 & 0.73 & 0.00 \\
\hline $105-645 \mathrm{E}-33 \mathrm{X}-5,20$ & 720.90 & 0.69 & 0.69 & 0.69 & 0.00 \\
\hline $105-645 \mathrm{E}-33 \mathrm{X}-5,40$ & 721.10 & 0.68 & 0.68 & 0.68 & 0.00 \\
\hline $105-645 \mathrm{E}-33 \mathrm{X}-5,60$ & 721.30 & 0.76 & 0.76 & 0.76 & 0.00 \\
\hline $105-645 \mathrm{E}-33 \mathrm{X}-5,80$ & 721.50 & 0.77 & 0.76 & 0.76 & 0.09 \\
\hline $105-645 \mathrm{E}-33 \mathrm{X}-5,100$ & 721.70 & 0.88 & 0.88 & 0.88 & 0.00 \\
\hline $105-645 \mathrm{E}-33 \mathrm{X}-5,120$ & 721.90 & 0.93 & 0.93 & 0.93 & 0.00 \\
\hline $105-645 \mathrm{E}-33 \mathrm{X}-5,141$ & 722.11 & 0.89 & 0.89 & 0.89 & 0.00 \\
\hline $105-645 \mathrm{E}-33 \mathrm{X}-6,10$ & 722.30 & 0.95 & 0.94 & 0.94 & 0.09 \\
\hline $105-645 \mathrm{E}-33 \mathrm{X}-6,28$ & 722.48 & 0.97 & 0.83 & 0.82 & 1.25 \\
\hline $105-645 \mathrm{E}-33 \mathrm{X}-6,50$ & 722.70 & 0.84 & 0.65 & 0.64 & 1.67 \\
\hline $105-645 \mathrm{E}-33 \mathrm{X}-6$ & 722.95 & 1.09 & 1.09 & 1.09 & 0.00 \\
\hline $105-645 \mathrm{E}-33 \mathrm{X}-6,95$ & 723.15 & 1.02 & 1.02 & 1.02 & 0.00 \\
\hline $105-645 \mathrm{E}-33 \mathrm{X}-6,115$ & 723.35 & 1.13 & 1.13 & 1.13 & 0.00 \\
\hline $105-645 \mathrm{E}-33 \mathrm{X}-6,135$ & 723.55 & 0.95 & 0.95 & 0.95 & 0.00 \\
\hline
\end{tabular}

Note: Records A, D, E, and F are shown in Fig. 6. $\mathrm{C}_{\text {org }}=$ total organic carbon; $\mathrm{CaCO}_{3}=$ carbonate content; $\mathrm{C}_{\mathrm{tot}}=$ total carbon; $\mathrm{C}_{\text {org }}{ }^{\prime}=$ wt $\%$ of carbonate-free fraction $=$ TOC $^{\prime}$ in Equation 1.

This indicates the immaturity of the organic matter, which is corroborated by the low temperatures of pyrolysis yield $\left(\mathrm{T}_{\max }\right)$ that range between $378^{\circ}$ and $435^{\circ} \mathrm{C}$ (Tables 3 and 6). In addition, we did not observe a significant increase in wavelength of maximum fluorescent intensity of sporinites.

\section{DISCUSSION}

\section{Depositional Environments in Baffin Bay}

The depositional regime at Site 645 is clearly dominated by the supply of both inorganic and organic terrigenous material throughout the entire sediment sequence, i.e., from early Miocene to Holocene time. Remains of planktonic biota are of only minor importance (Srivastava, Arthur, et al., 1987). The dominance of terrigenous organic components in the sediments at Site 645 is shown not only in the HI values (Figs. 10 and 11, Table 3), the $\mathrm{C} / \mathrm{N}$ ratios (Fig. 9), and the maceral composition (Fig. 11, Table 3), but also in the GC and GC/MS data (Ten Haven and Rullkötter, this volume). This dominance of the influx of terrigenous material is not surprising because Baffin Bay is a narrow, intracontinental basin near the surrounding continents
(Fig. 1). Thus, high amounts of (terrigenous) organic matter may have been deposited at Site 645. These amounts are distinctly higher than those recorded in open-marine environments, such as at Sites 646 and 647 (Figs. 6, 7, and 8; McIver, 1975; Müller and Suess, 1979). Because many of the terrestrial organic particles have diameters larger than $30 \mu \mathrm{m}$, especially in the younger sediments, we infer a near-continent deposition. At open-marine localities that are farther from continents or islands, terrestrial organic particles are usually distinctly smaller than $30 \mu \mathrm{m}$, if transportation and deposition by turbidity current activity can be excluded (Stein et al., 1988).

Higher accumulation rates of (dominantly terrigenous) organic carbon were observed in intervals between 18 and $12.5 \mathrm{Ma}$ and during the last 3.4 Ma in Baffin Bay (Fig. 9). This increased influx of terrigenous organic matter may have been caused by an increased fluvial sediment supply during that time. An increased fluvial influx is also supported by the high amounts of terrigenous clays recorded in the two intervals (Fig. 2). The increase of accumulation rates of the inorganic (i.e., carbonate and siliciclastic) and organic fractions recorded at 3.4 Ma coincides with the onset of major ice-rafted debris at Site 645 (Srivastava, Arthur, et al., 1987). During this time, the increased supply of terrigenous organic matter may have occurred during periods of increased inflow from melt waters from the surrounding continents. Because this increased supply of inorganic sediment fraction is distinctly higher than that of the organic components (Srivastava, Arthur, et al., 1987), the TOC percentage values decrease in the upper $400 \mathrm{~m}$ of the sequence (Fig. 6). This means that the decrease of TOC values is evidently caused by dilution from inorganic matter.

The interval of high influx of terrigenous organic matter in Baffin Bay during the last $3.4 \mathrm{Ma}$ is interrupted by two shortlived periods of distinctly lower accumulation rates. These minima occur at about $2 \mathrm{Ma}$ and between 0.3 and $0 \mathrm{Ma}$ (Fig. 9). During these time intervals, distinct maxima in $\delta^{18} \mathrm{O}$ indicate glacial maxima (e.g., Keigwin, 1979; Urban et al., 1983; Stein and Sarnthein, 1984). Thus, the fluvial supply may have been distinctly reduced, which may explain the reduced accumulation of terrigenous organic matter during that time.

The TOC data (Figs. 6 and 7) clearly show high-amplitude and short-term variations. The periods of these cyclic changes vary between about 20,000 and $100,000 \mathrm{yr}$ (Figs. 6 and 7). Thus. these periods may reflect Milankovitch-type climatic cycles. These short-term fluctuations are discussed in detail by Zimmerman et al. (unpublished data).

\section{Changes in Paleoproductivity in Baffin Bay and the Labrador Sea During Eocene to Holocene Time}

When interpreting the organic-carbon data in terms of oceanic paleoproductivity, one must separate the amount of terrigenous and marine organic carbon. Only the marine proportion of the organic-carbon content can be used to estimate paleoproductivity. We estimated the amounts of marine organic carbon from kerogen-microscopy data (Fig. 12; Stein et al., 1986). For the early Miocene and Pliocene to Pleistocene time intervals, the amounts of marine organic carbon were less than $10 \%$ of the TOC (or less than $0.25 \%$ of the whole sample). Only for the late/middle Miocene were higher marine organic-carbon values recorded; up to $50 \%$ of the TOC (or about $1 \%$ of the whole sample) may have been marine (Fig. 12). Surprisingly, this was not corroborated by the biological marker study of Ten Haven and Rullkötter (this volume).

Furthermore, to interpret marine organic-carbon values in terms of paleoproductivity, one must know whether the sediments were deposited under oxic or anoxic conditions (Müller and Suess, 1979; Bralower and Thierstein, 1984; Stein, 1986a, 1985 b). Because the preservation factor of organic matter is 
Table 3. Results from Rock-Eval pyrolysis and kerogen microscopy at Site 645.

\begin{tabular}{|c|c|c|c|c|c|c|c|c|c|c|c|c|}
\hline $\begin{array}{l}\text { Core/section } \\
\text { interval }(\mathrm{cm})\end{array}$ & $\begin{array}{l}\text { Depth } \\
\text { (mbsf) }\end{array}$ & $\begin{array}{c}\text { Age } \\
\text { (m.y.) }\end{array}$ & $\begin{array}{c}\text { TOC } \\
\text { (wt \%) }\end{array}$ & $\mathrm{HI}$ & $\mathrm{HI}^{*}$ & $\begin{array}{l}\mathrm{T}_{\max } \\
\left({ }^{\circ} \mathrm{C}\right)\end{array}$ & $\underset{(\%)}{G}$ & $\underset{(\%)}{H}$ & $\begin{array}{c}I \\
(\%)\end{array}$ & $\underset{(\%)}{J}$ & $\underset{(\%)}{K}$ & $\begin{array}{c}\mathrm{L} \\
(\%)\end{array}$ \\
\hline $105-645 B-2 X-4,7$ & 8.57 & 0.06 & 0.19 & & & & & & & & & \\
\hline $8 X-2,98$ & 64.18 & 0.44 & 0.24 & & & & & & & & & \\
\hline $14 X-2,37$ & 122.77 & 0.84 & 0.22 & & & & & & & & & \\
\hline $20 X-1,55$ & 179.75 & 1.23 & 0.80 & 10 & & 421 & 5 & 14 & 58 & 14 & 9 & \\
\hline $26 \mathrm{X}-1,120$ & 236.80 & 1.62 & 0.74 & 16 & & 410 & 9 & 13 & 58 & 14 & 6 & \\
\hline $30 \times-5,119$ & 281.39 & 1.98 & 0.81 & 28 & & 406 & 9 & 22 & 58 & 13 & & \\
\hline $32 X-1,143$ & 294.93 & 2.08 & 0.17 & & & & & & & & & \\
\hline $105-645 \mathrm{D}-8 \mathrm{R}-2,32$ & 342.22 & 2.46 & 0.69 & 5 & & 399 & & & 100 & & & \\
\hline $12 \mathrm{R}-2,04$ & 380.54 & 2.77 & 0.76 & 11 & & 423 & & & & & & \\
\hline $16 \mathrm{R}-1,99$ & 418.59 & 3.08 & 0.90 & 17 & & 421 & 3 & 3 & 69 & 11 & 14 & \\
\hline $16 \mathrm{R}-1,150$ & 419.10 & 3.08 & 0.72 & 7 & & 426 & & & & & & \\
\hline $18 \mathrm{R}-2,88$ & 439.28 & 3.25 & 0.67 & 40 & (167) & 403 & 0 & 5 & 33 & 14 & 48 & \\
\hline 105-645E-6R-5, 94 & 481.44 & 4.20 & 0.84 & 49 & & 413 & 3 & 8 & 81 & 5 & 3 & \\
\hline $8 R-5,21$ & 499.91 & 4.90 & 1.12 & 88 & & 409 & 3 & 17 & 66 & 10 & 3 & \\
\hline $9 \mathrm{R}-2,97$ & 505.87 & 5.10 & 1.00 & 102 & (275) & 415 & 52 & 4 & 17 & 13 & 13 & \\
\hline $11 \mathrm{R}-2,110$ & 525.20 & 5.70 & 0.78 & 51 & (176) & 428 & 14 & 14 & 23 & 9 & 41 & \\
\hline $13 \mathrm{R}-3,132$ & 546.12 & 6.50 & 0.77 & 35 & (136) & 390 & 6 & 13 & 9 & 13 & 50 & \\
\hline $15 \mathrm{R}-5,58$ & 567.68 & 7.20 & 1.50 & 85 & (295) & 414 & 37 & 21 & 18 & 13 & 5 & \\
\hline 16R-5, 99 & 577.79 & 7.60 & 1.08 & 106 & & 410 & 6 & 13 & 69 & 12 & 0 & \\
\hline $19 \mathrm{R}-4,92$ & 605.22 & 8.60 & 0.38 & 102 & & 413 & 7 & 29 & 50 & 14 & 0 & \\
\hline $24 \mathrm{R}-2,93$ & 640.43 & 9.49 & 0.71 & 5 & & 394 & & & & & & \\
\hline $26 \mathrm{R}-1,120$ & 658.30 & 9.86 & 0.96 & 7 & & 394 & 22 & 17 & 50 & 11 & 0 & \\
\hline $28 \mathrm{R}-1,26$ & 676.76 & 10.23 & 0.65 & 11 & & 402 & & & & & & \\
\hline $30 \mathrm{R}-1,145$ & 697.05 & 10.66 & 1.06 & 32 & & 402 & 18 & 19 & 46 & 14 & 4 & \\
\hline $32 \mathrm{R}-2,130$ & 714.50 & 11.02 & 1.15 & 35 & & 406 & 11 & 4 & 56 & 19 & 11 & \\
\hline $35 \mathrm{R}-4,15$ & 738.65 & 11.52 & 1.11 & 112 & & 409 & 29 & 43 & 28 & 0 & 0 & \\
\hline $36 \mathrm{R}-1,120$ & 744.90 & 11.65 & 1.20 & 142 & & 419 & 33 & 17 & 33 & 17 & 0 & 0.26 \\
\hline $37 R-5,63$ & 760.03 & 11.98 & 1.08 & 105 & & 418 & 29 & 24 & 42 & 2 & 2 & \\
\hline $38 \mathrm{R}-3,47$ & 766.47 & 12.09 & 0.99 & 95 & & 416 & 40 & 24 & 32 & 4 & 0 & \\
\hline $39 \mathrm{R}-4,134$ & 778.14 & 12.33 & 1.11 & 116 & & 423 & 4 & 10 & 68 & 14 & 4 & \\
\hline $40 \mathrm{R}-4,93$ & 788.03 & 12.59 & 1.16 & 101 & & 415 & 13 & 30 & 43 & 9 & 4 & \\
\hline $41 R-2,70$ & 794.20 & 12.69 & 1.59 & 130 & & 427 & 40 & 13 & 33 & 7 & 7 & 0.31 \\
\hline $43 R-1,00$ & 811.20 & 13.00 & 2.15 & & & & 27 & 9 & 37 & 27 & 0 & 0.26 \\
\hline $44 \mathrm{R}-2,00$ & 823.30 & 13.28 & 1.79 & 148 & & 429 & 39 & 10 & 41 & 10 & 0 & 0.30 \\
\hline $49 \mathrm{R}-5,80$ & 875.40 & 14.37 & 2.29 & 217 & & 422 & 12 & 23 & 56 & 5 & 4 & \\
\hline $49 \mathrm{R}-5,140$ & 876.00 & 14.39 & 1.61 & 170 & & 430 & 27 & 18 & 28 & 27 & 0 & 0.27 \\
\hline $50 \mathrm{R}-1,150$ & 879.60 & 14.49 & 2.01 & 144 & & 429 & 13 & 20 & 37 & 23 & 7 & 0.26 \\
\hline $52 \mathrm{R}-2,62$ & 890.02 & 14.68 & 2.98 & 225 & & 430 & 25 & 17 & 41 & 17 & 0 & 0.27 \\
\hline $54 \mathrm{R}-2,76$ & 909.46 & 15.07 & 2.30 & 159 & & 430 & 13 & 22 & 52 & 13 & 0 & 0.29 \\
\hline $54 R-6,122$ & 915.92 & 15.20 & 1.36 & 119 & & 418 & 4 & 33 & 47 & 13 & 2 & \\
\hline $56 \mathrm{R}-4,140$ & 932.50 & 15.57 & 1.46 & 138 & & 428 & 17 & 3 & 66 & 10 & 0 & 0.25 \\
\hline $57 \mathrm{R}-4,99$ & 941.69 & 15.70 & 0.83 & 84 & & 413 & 0 & 10 & 60 & 30 & 0 & \\
\hline $60 \mathrm{R}-1,150$ & 966.80 & 16.27 & 1.70 & 115 & & 429 & 27 & 18 & 28 & 27 & 0 & 0.28 \\
\hline $62 R-5,140$ & 991.90 & 16.77 & 1.83 & 120 & & 427 & 7 & 14 & 57 & 14 & 4 & 0.30 \\
\hline $62 \mathrm{R}-6,37$ & 992.37 & 16.78 & 1.44 & 95 & & 420 & 5 & 5 & 90 & 0 & 0 & \\
\hline $64 R-5,94$ & 1010.84 & 17.20 & 1.47 & 88 & & 421 & 0 & 15 & 63 & 18 & 4 & \\
\hline $66 \mathrm{R}-2,89$ & 1025.69 & 17.49 & 1.04 & & & & 13 & 8 & 62 & 17 & 0 & 0.26 \\
\hline $67 \mathrm{R}-5,120$ & 1040.10 & 17.80 & 1.00 & 84 & & 413 & 0 & 5 & 90 & 5 & 0 & \\
\hline $71 \mathrm{R}-4,133$ & 1076.93 & 18.20 & 0.81 & 80 & & 407 & 10 & 23 & 49 & 19 & 0 & \\
\hline $74 \mathrm{R}-5,110$ & 1106.90 & 19.10 & 0.57 & 82 & & 407 & 0 & 33 & 51 & 17 & 0 & \\
\hline $77 R-5,100$ & 1135.20 & 19.75 & 1.09 & 92 & & 415 & 8 & 4 & 81 & 8 & 0 & \\
\hline $78 \mathrm{R}-1,41$ & 1138.31 & 19.80 & 0.66 & 113 & & 419 & 0 & 21 & 79 & 0 & 0 & \\
\hline
\end{tabular}

$\mathrm{HI}=$ hydrogen index in $\mathrm{mg} \mathrm{HC} / \mathrm{g} \mathrm{C}_{\text {org }}$, measured on bulk-sediment samples; $\mathrm{HI}^{*}=$ hydrogen index in $\mathrm{mg} \mathrm{HC} \mathrm{g} \mathrm{C}$ org' measured on kerogen concentrates; TOC $=$ total organic carbon; $\mathrm{T}_{\max }=$ temperature of maximum pyrolysis yield in ${ }^{\circ} \mathrm{C} ; \mathrm{G}=$ alginite; $\mathrm{H}=$ liptodetrinite; $\mathrm{I}=$ vitrinite; $\mathrm{J}=$ sporinite; $\mathrm{K}=$ inertinite; $\mathrm{L}=$ vitrinite reflectance values. Numbers in columns $\mathrm{G}-\mathrm{K}$ are percentage values of the total macerals.

very high under anoxic conditions (i.e., much higher than under oxic conditions), organic-carbon-rich sediments can be deposited in this environment, although oceanic productivity may be very low. At Sites 645,646 , and 647 , anoxic conditions are unlikely because of bioturbation recorded in the entire sediment sequence (Srivastava, Arthur, et al., 1987). An oxic depositional environment is also supported by the relationship between organic-carbon content and sedimentation rate (Fig. 13; Stein, 1986b). Thus, oxic environments may have occurred in Baffin Bay and Labrador Sea throughout the time intervals discussed here.

In oxic environments, paleoproductivity may be roughly estimated from the comparison of (marine) organic-carbon content and sedimentation rate (Fig. 13; Müller and Suess, 1979; Stein, 1986b). From this, relatively low paleoproductivity was typical at all three sites during most of the time intervals discussed here.

At Site 645 , the paleoproductivity was very low during the early Miocene and the early Pliocene, whereas during late/middle Miocene time some higher oceanic productivity may have been possible (Fig. 13). The late Pliocene/Pleistocene data (Fig. 13) fall out of the trend, probably from dilution effects of icerafted debris (Srivastava, Arthur, et al., 1987).

At the Labrador Sea Site 646, paleoproductivity was probably very low during the late Miocene. A slight increase in productivity probably occurred during latest Miocene and Pliocene-Pleistocene time (Fig. 13).

At Site 647 , productivity was very low during middle Eocene and (middle) Miocene time. During the late Eocene/early Oligocene and late Pliocene/Pleistocene, higher productivity probably occurred (Fig. 13). Higher productivity during the late Eo- 
cene/early Oliogocene also is supported by a major occurrence of biogenic opal and opal-CT at Site 647 (Fig. 8; Bohrmann and Stein, this volume; Stax, 1988).

According to the data shown in Figure 13, the surface-water productivity estimated for Sites 645,646 , and 647 , was distinctly lower than that observed in typical high-productivity areas (field $A$ ' in Fig. 13). However, an unusually high oxidation rate of organic matter from the cold oxygen-rich water masses may have resulted in an unusually high loss of marine organic carbon in polar and subpolar regions. In this case, the estimated paleoproductivity values are too low and must be considered as minimum values. At Sites 646 and 647, Rock-Eval data support the dominance of highly oxidized organic matter (Table 6; T. Cederberg, pers. comm., 1987) This may also explain why the data from Sites 645, 646, and 647 shown in Figure 13 fall below trend line $A$, which is based on data derived from tropical, subtropical, and temperate areas (Stein, 1986b). For oxic environments in polar and subpolar regions, trend line $A$ may have a lower slope. However, this hypothesis must be tested with data from Holocene polar and subpolar sediments.

To obtain more quantitative estimates of paleoproductivity, models and equations are available to calculate productivity values of the overlying surface-water mass from sediment data (see "Methods" section, this chapter; Müller and Suess, 1979; Bralower and Thierstein, 1984; Stein, 1986a; Sarnthein et al., 1987).

Average paleoproductivity values were obtained using Equation 4 (Stein, 1986a; summarized in Table 7). At Site 645, most of the Neogene time interval is characterized by very low surface-water productivity (mean values between 40 and $90 \mathrm{gC}$. $\mathrm{m}^{-2} \cdot \mathrm{k} . \mathrm{y} .^{-1}$, Table 7). This means that productivity was similar to or less than that measured in Baffin Bay today (Fig. 14; Romankevich, 1984). Only in the middle Miocene may higher oceanic productivity have occurred in Baffin Bay. Productivity may have reached values of almost 150 , and possibly up to $200 \mathrm{gC}$. $\mathrm{m}^{-2} \cdot$ k.y. ${ }^{-1}$ (Fig. 14, Table 7).

The intervals of increased productivity at Site 645 coincide with intervals of increased clay content (Fig. 2). This may indicate that a fluvial nutrient supply may have caused the increased productivity during that time (Church, 1970). However, the inflow of cold nutrient-rich water masses (from arctic areas ?) or local upwelling activities cannot be excluded as causes for the higher fertility of surface water in western Baffin Bay. According to Williams (1986), it is likely that the western side of Baffin Bay has a relatively high surface-water productivity at least seasonally, as today, the Baffin Island Current sweeps along the fast ice edge and causes upwelling.

At the Labrador Sea Sites 646 and 647, paleoproductivity values range between 100 and $170 \mathrm{gC} \cdot \mathrm{m}^{-2} \cdot \mathrm{k} \cdot \mathrm{y} \cdot{ }^{-1}$ and between 40 and $160 \mathrm{gC} \cdot \mathrm{m}^{-2} \cdot \mathrm{k} . \mathrm{y}^{-1}$, respectively (Table 7). During late Eocene/early Oliogocene and late Pliocene to Pleistocene time, values of 90 to $170 \mathrm{gC} \cdot \mathrm{m}^{-2} \cdot \mathrm{k} . \mathrm{y}^{-1}$ were typical, i.e., values that are similar to those measured today in the Labrador Sea (Romankevich, 1984). The lower values of 40 to 70 were estimated for early Eocene and (middle) Miocene time (Table 7). The increase in productivity during Eocene/early Oligocene time, which is also indicated in the biogenic opal and opalCT contents (Bohrmann and Stein, this volume; Stax, 1988), was accompanied by a cooling of surface waters (Srivastava, Arthur, et al., 1987). These colder and probably more nutrient-rich water masses may have caused the increased paleoproductivity during that time.

\section{CONCLUSIONS}

The results of our detailed organic geochemical and petrographic investigations can be summarized as follows.

1. The Neogene sediments of Site 645 are characterized by relatively high organic-carbon values, most of which range between $0.5 \%$ and almost $3 \%$. Maximum values are concentrated in the middle Miocene time interval. At Sites 646 and 647, organic-carbon contents are distinctly lower and vary between $0.1 \%$ and $0.75 \%$.

2. The organic matter deposited at Site 645 is dominated by terrigenous components (i.e., mainly vitrinites and sporinites). Only in the late/middle Miocene were higher amounts of marine organic matter recorded. At open-ocean Sites 646 and 647, a predominance of marine organic carbon is suggested.

3. At Site 645 , surface-water productivity was relatively low (i.e., similar or less than that measured in Baffin Bay today) during most parts of the Neogene time interval. Only in the late/middle Miocene may higher values of up to 150 (200) $\mathrm{gC}$. $\mathrm{m}^{-2} \cdot \mathrm{k} . \mathrm{y} .^{-1}$ have been reached. At Sites 646 and 647, estimated mean paleoproductivity values vary between 40 and $170 \mathrm{gC}$. $\mathrm{m}^{-2} \cdot \mathrm{k} \cdot \mathrm{y} .^{-1}$.

4. Vitrinite reflectance values of less than $0.31 \%$ indicate that the organic matter is immature.

\section{ACKNOWLEDGMENTS}

We thank W. Benders, E. Biermanns, V. Lukas, D. Ortmann, H. Schnitzler, and K. Sellinghof for technical assistance. We thank T. J. Bralower and P. A. Meyers for their constructive suggestions for improving the manuscript. Financial support by the Deutsche Forschungsgemeinschaft (Grants No. STE $412 / 1$ and No. WE 346/27) is gratefully acknowledged.

\section{REFERENCES}

Betzer, P. R., Showers, W. J., and Laws, E. A., 1984. Primary productivity and particle fluxes on a transect of the equator at $153^{\circ} \mathrm{W}$ in the Pacific Ocean. Deep-Sea Res., 131:1-11.

Bordowskiy, O. K., 1965a. Sources of organic matter in marine basins. Mar. Geol., 3:5-31.

1965b. Accumulation of organic matter in bottom sediments. Mar. Geol., 3:33-82.

Bralower, T. J., and Thierstein, H. R., 1984. Low productivity and slow deep-water circulation in mid-Cretaceous oceans. Geology, 12:614618.

Church, M. A., 1970. Baffin Island Sandur. A study of arctic fluvial environments [M. A. thesis]. Vancouver, University of British Columbia.

Durand, B., 1980. Kerogen: Paris (Editions Technip), 1-519.

Emery, K. O., and Uchupi, E., 1984. The Geology of the Atlantic Ocean: New York (Springer-Verlag).

Espitalié, J., Laporte, J. L., Madec, M., Marquis, F., Leplat, P., Paulet, J., and Boutefeu, A., 1977. Méthode rapide de charactérisation des roches-mére, de leur potential pétrolier et de leur dégre d'évolution. Rev. Inst. Français Petrol., 32:23-42.

Hutton, A. C., Kantsler, A. J., Cook, A. C., and McKirdy, D. M., 1980. Organic matter in oil shales. Austr. Pet. Expl. Assoc., 20:4467.

Keigwin, L. D., Jr., 1979. Late Cenozoic stable isotope stratigraphy and paleoceanography of DSDP sites from the east equatorial and north central Pacific Ocean. Earth Plan. Sci. Lett., 45:361-382.

Littke, R., 1987. Petrology and genesis of upper carboniferous seams from the Ruhr region, West Germany. Int. J. Coal Geol., 7:147-184.

Marko, J. R., Birch, J. R., and Wilson, M. A., 1982. A study of longterm satellite-tracked iceberg drifts in Baffin Bay and Davis Strait. Arctic, 35:234-240.

McIver, R., 1975. Hydrocarbon occurrence from JOIDES Deep Sea Drilling Project. Proc. Ninth Pet. Congr., 269-280.

Müller, P. J., 1977. C/N ratios in Pacific deep-sea sediments: Effect of inorganic ammonium and organic nitrogen compounds sorbed by clays. Geochim. Cosmochim. Acta, 41:765-776.

Müller, R., and Suess, E., 1979. Productivity, sedimentation rate, and sedimentary organic carbon content in the oceans. Part I. organic carbon preservation. Deep-Sea Res., 26(A):1347-1362.

Raiswell, R., and Berner, R. A., 1987. Organic carbon losses during the burial and the thermal maturation of normal marine shales. Terra Cognita, 7:195. 
Romankevich, E. A., 1984. Geochemistry of Organic Matter in the Ocean: Berlin-Heidelberg (Springer-Verlag).

Sarnthein, M., Winn, K., and Zahn, R., 1987. Paleoproductivity of oceanic upwelling and the effect on atmospheric $\mathrm{CO}_{2}$ and climatic change during deglaciation times. In Berger, W., and Labeyrie, L. (Eds.), Proc. Workshop Abrupt Climatic Change, Nato/NSF A.R.W., Biviers, 1985.

Scheffer, F., and Schachtschabel, P., 1984. Lehrbuch der Bodenkunde: Stuttgart (F. Enke Verlag).

Srivastava, S., Arthur, M. A., et al., 1987. Proc. ODP, Init. Repts., 105: College Station, TX (Ocean Drilling Program).

Srivastava, S., Falconer, R.K.H., and MacLean, B., 1981. Labrador Sea, Davis Strait, Baffin Bay: geology and geophysics-a review. In Kerr, J. W., Fergusson, A. J., and Machan, L. C.(Eds.), Geology of the North Atlantic Borderlands, Can. Soc. Pet. Geol. Mem., 8:333398.

Stach, E., Mackowsky, M.-Th., Teichmüller, M., Taylor, G. H., Chandra, D., and Teichmüller, R., 1982. Stach's Textbook of Coal Petrology: Berlin (Borntraeger-Verlag).

Stax, Rainer, 1988. Paläoenvironment-Rekonstruktionen nach mineralogischen, granulometrischen und C-org-Untersuchungen an quartären und tertiären Sedimenten von ODP-Site 647, Labrador See, [Unpublished M. S. thesis]. Giessen, University of Giessen.

Stein, R., 1986a. Surface-water paleoproductivity as inferred from sediments deposited in oxic and anoxic deep-water environments of the Mesozoic Atlantic Ocean. In Degens, E. T., Brassell, S., and Meyers, P.(Eds.), Proc. Black Shale Conf., Hamburg, 1985, Mitt. Geol.Pal. Inst. Hamburg, 60:55-70.

, 1986b. Organic carbon and sedimentation rate-Further evidence for anoxic deep-water conditions in the Cenomanian/Turonian Atlantic Ocean. Mar. Geol., 72:199-209.
Stein, R., Rullkötter, J., and Welte, D., 1986. Accumulation of organiccarbon-rich sediments in the Late Jurassic and Cretaceous Atlantic Ocean-a synthesis. Chem. Geol., 56:1-32.

Stein, R., Rullkötter, J., Littke, R., Schaefer, R. G., and Welte, D. H., 1988. Organofacies reconstruction and lipid geochemistry of sediments from the Galicia margin, Northeast Atlantic (ODP Leg 103). In Boillot, G., Winterer, E. L., et al., Proc. ODP, Sci. Results, 103: College Station, TX (Ocean Drilling Program).

Stein, R., and Sarnthein, M., 1984. Late Neogene events of atmospheric and oceanic circulation offshore Northwest Africa: high-resolution record from deep-sea sediments. Palaeoecology Africa, 16:9-36.

Suess, E., 1980. Particulate organic carbon flux in the ocean-surface productivity and oxygen utilization. Nature, 288:260-263.

Tissot, B., and Welte, D. H., 1984. Petroleum Formation and Occurence: Berlin-Heidelberg (Springer-Verlag).

Tchernia, P., 1982. Descriptive Regional Oceanography, Pergamon Marine Series, Vol. 3: Oxford (Pergamon).

Urban, B., Arias, C., Bigazzi, G., and Bonadonna, F., 1983. Early Pleistocene palynostratigraphy of Fornace, Tini, Vale Ricca (Central Italy). Palaeogeogr., Palaeoclimatol., Palaeoecol., 41:153-164.

Van Andel, Tj. H., Heath, G. R., and Moore, T. C., 1975. Cenozoic history and paleoceanography of the central equatorial Pacific. Geol. Soc. Am., Mem. 143.

Williams, K. M., 1986. Recent arctic marine diatom assemblages from bottom sediments in Baffin Bay and Davis Strait. Mar. Micropalentol., 10:327-342.

Date of initial receipt: 23 June 1987

Date of acceptance: 1 December 1987

Ms 105B-154 


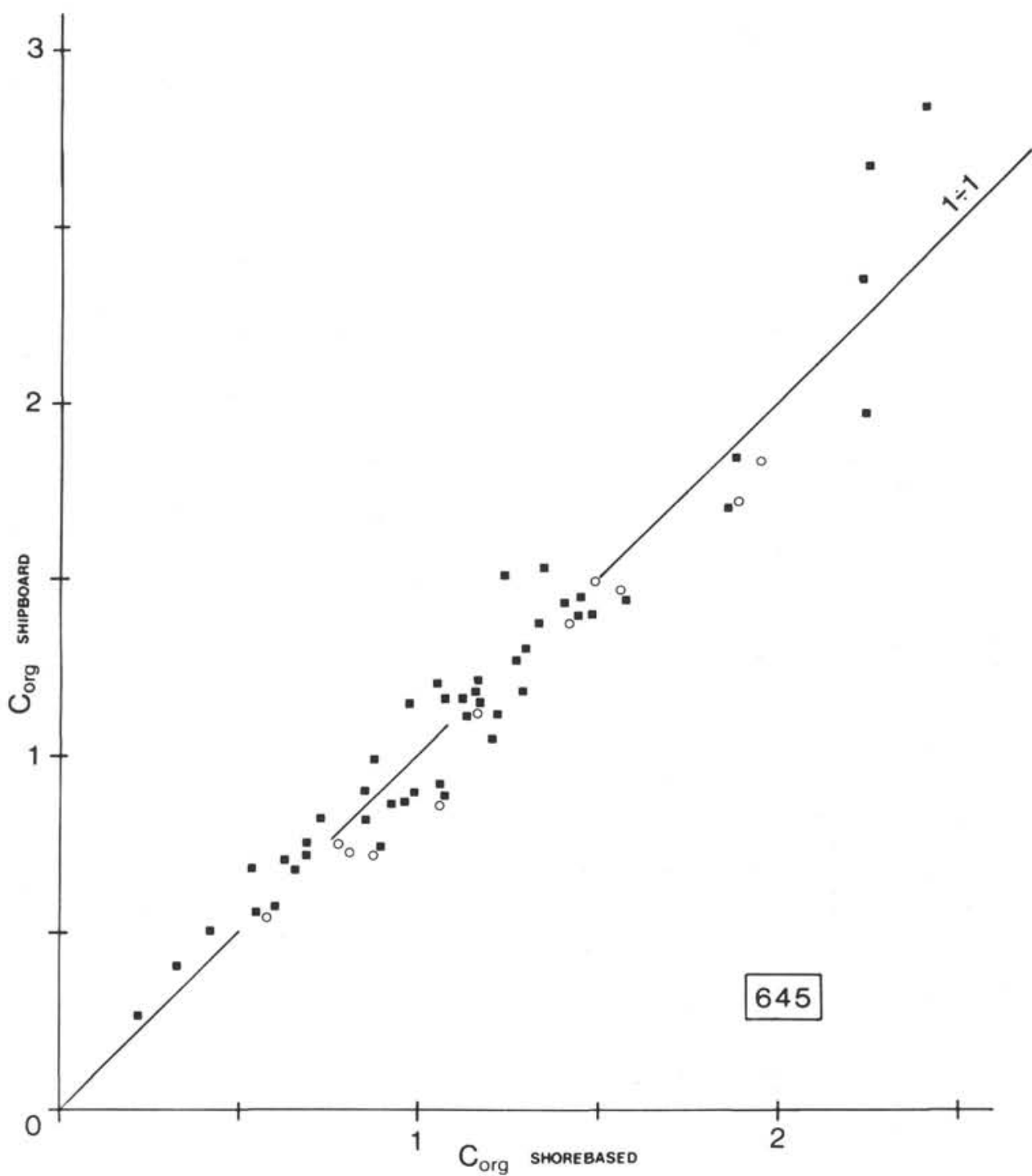

Figure 3. Comparison of total organic carbon values (in wt \%) determined on board JOIDES Resolution using a $\mathrm{CHN}$ analyzer, with values determined in our laboratory using a LECO (solid quadrants) or CHN analyzer (open circles). 
Table 4. Total carbon, total organic carbon, carbonate carbon, and $\mathrm{C} / \mathrm{N}$ ratios at Site 646 .

\begin{tabular}{|c|c|c|c|c|c|c|}
\hline $\begin{array}{l}\text { Core/section } \\
\text { interval }(\mathrm{cm})\end{array}$ & $\begin{array}{l}\text { Depth } \\
\text { (mbsf) }\end{array}$ & $\mathrm{C}_{\text {tot }}$ & $\mathrm{C}_{\text {org }}{ }^{\prime}$ & $\mathrm{C}_{\text {org }}$ & $\mathrm{CaCO}_{3}$ & $\mathrm{C} / \mathrm{N}$ \\
\hline \multicolumn{7}{|l|}{ A } \\
\hline $105-646 A-1-2,68$ & 2.18 & 0.79 & 0.27 & 0.26 & 4.4 & \\
\hline $2-3,45$ & 7.45 & 1.62 & 0.17 & 0.15 & 12.3 & \\
\hline $3-3,68$ & 20.18 & 1.28 & 0.20 & 0.18 & 9.2 & \\
\hline $4-5,111$ & 33.31 & 0.54 & 0.24 & 0.23 & 2.6 & \\
\hline $5-6,86$ & 44.16 & 4.03 & 0.25 & 0.17 & 32.2 & \\
\hline $6-2,08$ & 47.08 & 4.93 & 0.36 & 0.22 & 39.3 & \\
\hline $6-2,21$ & 47.21 & 0.58 & 0.39 & 0.38 & 1.6 & \\
\hline $6-4,119$ & 51.19 & 4.98 & 0.26 & 0.16 & 40.2 & \\
\hline $7-5,7$ & 61.17 & 0.70 & 0.21 & 0.20 & 4.2 & \\
\hline $7-5,140$ & 62.50 & 3.14 & 0.18 & 0.13 & 25.0 & \\
\hline $7-6,105$ & 63.65 & 2.53 & 0.19 & 0.15 & 19.8 & \\
\hline $8-2,45$ & 66.75 & 2.75 & 0.34 & 0.27 & 20.7 & \\
\hline $9-1,79$ & 75.19 & 1.73 & & & & \\
\hline $9-2,79$ & 76.69 & 0.59 & 0.20 & 0.19 & 3.3 & \\
\hline $9-3,80$ & 78.20 & 3.68 & 0.29 & 0.21 & 29.0 & \\
\hline $9-4,79$ & 79.69 & 1.05 & 0.45 & 0.43 & 5.2 & \\
\hline $9-5,80$ & 81.20 & 1.76 & 0.33 & 0.29 & 12.3 & \\
\hline $10-5,129$ & 91.39 & 5.26 & 0.76 & 0.46 & 40.0 & \\
\hline $10-5,139$ & 91.49 & 0.85 & 0.18 & 0.17 & 5.7 & \\
\hline $11-6,94$ & 102,24 & 0.93 & 0.26 & 0.25 & 5.7 & \\
\hline $105-646 \mathrm{~B}-12-5,53$ & 107.93 & 3.80 & 0.41 & 0.29 & 29.3 & 14 \\
\hline $12-5,61$ & 108.01 & 5.18 & 0.75 & 0.45 & 39.4 & 25 \\
\hline $12-5,70$ & 108.10 & 1.48 & 0.25 & 0.22 & 10.5 & 3 \\
\hline $14-4,81$ & 126.11 & 0.59 & 0.29 & 0.28 & 2.6 & 10 \\
\hline $18-2,58$ & 161.18 & 0.60 & 0.26 & 0.25 & 2.9 & 4 \\
\hline $20-3,48$ & 181.98 & 0.47 & 0.35 & 0.35 & 1.0 & 12 \\
\hline $21-4,46$ & 193.16 & 0.58 & 0.58 & 0.58 & 0.0 & 14 \\
\hline $22-1,138$ & 199.18 & 0.76 & 0.76 & 0.76 & 0.0 & 19 \\
\hline $23-2,110$ & 210.10 & 1.77 & 0.26 & 0.23 & 12.9 & 4 \\
\hline $26-4,107$ & 241.67 & 0.55 & 0.34 & 0.34 & 1.8 & 17 \\
\hline $30-4,92$ & 280.42 & 0.47 & 0.45 & 0.45 & 0.2 & 15 \\
\hline $31-4,70$ & 289.90 & 1.27 & 0.47 & 0.44 & 6.9 & 12 \\
\hline $32-2,132$ & 297.22 & 0.51 & 0.51 & 0.51 & 0.0 & 13 \\
\hline $33-5,95$ & 311.05 & 1.42 & 0.57 & 0.53 & 7.4 & 14 \\
\hline $35-1,97$ & 324.37 & 0.70 & 0.42 & 0.41 & 2.4 & 6 \\
\hline $36-6,37$ & 340.97 & 0.47 & 0.46 & 0.46 & 0.1 & 12 \\
\hline $37-6,90$ & 351.20 & 3.25 & 0.39 & 0.29 & 24.6 & 10 \\
\hline $38-1,27$ & 352.67 & 1.09 & 0.25 & 0.23 & 7.1 & 8 \\
\hline $38-3,105$ & 356.45 & 0.76 & 0.39 & 0.38 & 3.2 & 13 \\
\hline $41-3,129$ & 385.59 & 2.54 & 0.63 & 0.52 & 16.8 & 13 \\
\hline $43-3,118$ & 404.88 & & 0.44 & & & 9 \\
\hline $46-1,10$ & 429.90 & 1.45 & 0.42 & 0.38 & 8.9 & 8 \\
\hline $49-2,103$ & 461.33 & 3.04 & 0.42 & 0.32 & 22.6 & 11 \\
\hline $50-2,58$ & 470.58 & 3.13 & 0.47 & 0.36 & 23.1 & 9 \\
\hline $51-2,15$ & 479.75 & 0.37 & 0.36 & 0.36 & 0.1 & 12 \\
\hline $56-2,12$ & 527.82 & 0.98 & 0.39 & 0.37 & 5.1 & 8 \\
\hline $58-2,32$ & 547.32 & 0.56 & 0.52 & 0.52 & 0.3 & 10 \\
\hline $60-2,6$ & 565.96 & 0.36 & 0.35 & 0.35 & 0.1 & 12 \\
\hline $61-3,12$ & 577.12 & 0.45 & 0.45 & 0.45 & 0.0 & 11 \\
\hline $62-2,32$ & 585.42 & 1.66 & 0.38 & 0.34 & 11.0 & 13 \\
\hline $63-1,118$ & 594.38 & 1.34 & 0.32 & 0.29 & 8.7 & 11 \\
\hline $65-4,57$ & 617.57 & 1.59 & 0.36 & 0.32 & 10.6 & 7 \\
\hline $66-1,131$ & 623.51 & 2.16 & 0.38 & 0.32 & 15.3 & 8 \\
\hline $67-1,85$ & 632.65 & 0.38 & 0.32 & 0.32 & 0.5 & 8 \\
\hline $68-3,33$ & 644.63 & 0.41 & 0.37 & 0.37 & 0.3 & 12 \\
\hline $69-1,84$ & 651.74 & 2.22 & 0.33 & 0.28 & 16.2 & 7 \\
\hline $70-1,71$ & 661.21 & 1.48 & 0.41 & 0.37 & 9.2 & 7 \\
\hline $71-1,58$ & 670.78 & 0.84 & 0.31 & 0.30 & 4.5 & 8 \\
\hline $71-1,69$ & 670.89 & 7.45 & 0.70 & 0.28 & 59.7 & 12 \\
\hline $72-2,32$ & 681,52 & 2.53 & 0.42 & 0.34 & 18.2 & 14 \\
\hline $73-1,76$ & 690.06 & 2.84 & 0.3 & 0.23 & 21.7 & 6 \\
\hline $75-3,87$ & 712.47 & 0.29 & 0.27 & 0.27 & 0.2 & 7 \\
\hline $76-2,72$ & 720.52 & 0.32 & 0.24 & 0.24 & 0.7 & 8 \\
\hline $77-4,42$ & 732.82 & 0.66 & 0.29 & 0.28 & 3.2 & 10 \\
\hline $78-1,82$ & 738.52 & 0.59 & 0.34 & 0.33 & 2.1 & 7 \\
\hline $79-1,59$ & 747.99 & 0.27 & 0.22 & 0.22 & 0.4 & 6 \\
\hline $80-1,104$ & 758.04 & 1.62 & 0.34 & 0.30 & 11.0 & 9 \\
\hline \multicolumn{7}{|l|}{ B } \\
\hline $105-646 \mathrm{~A}-2-1,30$ & 5.30 & 1.57 & 0.31 & 0.28 & 10.78 & \\
\hline $2-1,50$ & 5.50 & 3.87 & 0.34 & 0.24 & 30.28 & \\
\hline $2-1,70$ & 5.70 & 1.12 & 0.31 & 0.29 & 6.93 & \\
\hline $2-1,90$ & 5.90 & 0.54 & 0.33 & 0.32 & 1.80 & \\
\hline $2-1,111$ & 6.11 & 0.90 & 0.34 & 0.32 & 4.80 & \\
\hline
\end{tabular}

Table 4 (continued).

\begin{tabular}{|c|c|c|c|c|c|c|}
\hline $\begin{array}{l}\text { Core/section } \\
\text { interval }(\mathrm{cm})\end{array}$ & $\begin{array}{l}\text { Depth } \\
\text { (mbsf) }\end{array}$ & $\mathrm{C}_{\text {tot }}$ & $\mathrm{C}_{\text {org }}$ & $\mathrm{C}_{\text {org }}$ & $\mathrm{CaCO}_{3}$ & $\mathrm{C} / \mathrm{N}$ \\
\hline \multicolumn{7}{|l|}{ B } \\
\hline $2-1,132$ & 6.32 & 0.93 & 0.17 & 0.16 & 6.42 & \\
\hline $2-2,0$ & 6.50 & 1.05 & 0.19 & 0.18 & 7.28 & \\
\hline $2-2,21$ & 6.71 & 1.17 & 0.25 & 0.23 & 7.83 & \\
\hline $2-2,41$ & 6.91 & 1.03 & 0.24 & 0.22 & 6.72 & \\
\hline $2-2,61$ & 7.11 & 0.93 & 0.22 & 0.21 & 6.03 & \\
\hline $2-2,80$ & 7.30 & 0.38 & 0.10 & 0.10 & 2.35 & \\
\hline $2-2,100$ & 7.50 & 0.62 & 0.22 & 0.21 & 3.40 & \\
\hline $2-2,121$ & 7.71 & 0.64 & 0.18 & 0.17 & 3.89 & \\
\hline $2-2,142$ & 7.92 & 0.33 & 0.15 & 0.14 & 9.96 & \\
\hline $2-3,10$ & 8.10 & 1.27 & 0.18 & 0.16 & 9.22 & \\
\hline $2-3,30$ & 8.30 & 2.01 & 0.19 & 0.16 & 15.41 & \\
\hline $2-3,50$ & 8.50 & 1.49 & 0.22 & 0.20 & 10.78 & \\
\hline $2-3,69$ & 8.69 & 1.32 & 0.26 & 0.24 & 9.03 & \\
\hline $2-3,90$ & 8.90 & 0.58 & 0.26 & 0.25 & 2.73 & \\
\hline $2-3,110$ & 9.10 & 0.86 & 0.14 & 0.13 & 6.07 & \\
\hline $2-3,130$ & 9.30 & 1.03 & 0.20 & 0.19 & 7.03 & \\
\hline $2-4,0$ & 9.50 & 1.16 & 0.19 & 0.17 & 8.21 & \\
\hline $2-4,20$ & 9.70 & 1.88 & 0.22 & 0.19 & 14.09 & \\
\hline $2-4,40$ & 9.90 & 1.44 & 0.23 & 0.21 & 10.28 & \\
\hline $2-4,60$ & 10.10 & 0.45 & 0.19 & 0.19 & 2.20 & \\
\hline $2-4,80$ & 10.30 & 1.20 & 0.29 & 0.27 & 7.77 & \\
\hline $2-4,100$ & 10.50 & 0.36 & 0.24 & 0.24 & 1.02 & \\
\hline $2-4,120$ & 10.70 & 0.36 & 0.30 & 0.30 & 0.51 & \\
\hline $2-4,140$ & 10.90 & 0.41 & 0.38 & 0.38 & 0.26 & \\
\hline $2-5,10$ & 11.10 & 0.43 & 0.38 & 0.38 & 0.43 & \\
\hline $2-5,30$ & 11.30 & 0.51 & 0.43 & 0.43 & 0.69 & \\
\hline $2-5,50$ & 11.50 & 0.88 & 0.48 & 0.46 & 3.47 & \\
\hline $2-5,70$ & 11.70 & 0.93 & 0.44 & 0.42 & 4.24 & \\
\hline $2-5,90$ & 11.90 & 0.78 & 0.41 & 0.40 & 3.19 & \\
\hline $2-5,110$ & 12.10 & 0.97 & 0.40 & 0.38 & 4.91 & \\
\hline $2-6,0$ & 12.50 & 0.97 & 0.31 & 0.29 & 5.65 & \\
\hline $2-6,21$ & 12.71 & 0.98 & 0.32 & 0.30 & 5.65 & \\
\hline $2-6,39$ & 12.89 & 0.79 & 0.45 & 0.40 & 11.60 & \\
\hline $2-6,60$ & 13.10 & 0.64 & 0.31 & 0.30 & 2.82 & \\
\hline $2-6,80$ & 13.30 & 0.88 & 0.27 & 0.26 & 5.20 & \\
\hline $2-6,100$ & 13.50 & 0.57 & 0.31 & 0.30 & 2.22 & \\
\hline $2-6,120$ & 13.70 & 0.33 & 0.17 & 0.17 & 1.35 & \\
\hline $2-6,143$ & 13.93 & 1.10 & 0.43 & 0.41 & 5.79 & \\
\hline \multicolumn{7}{|l|}{$\mathrm{C}$} \\
\hline $105-646 \mathrm{~A}-7-2,110$ & 57.70 & 0.48 & 0.27 & 0.27 & 1.79 & \\
\hline $7-2,130$ & 57.90 & 0.71 & 0.22 & 0.21 & 4.16 & \\
\hline $7-2,150$ & 58.10 & 0.46 & 0.25 & 0.25 & 1.79 & \\
\hline $7-3,18$ & 58.28 & 0.99 & 0.17 & 0.16 & 6.93 & \\
\hline $7-3,40$ & 58.50 & 0.70 & 0.16 & 0.15 & 4.56 & \\
\hline $7-3,62$ & 58.72 & 0.70 & 0.20 & 0.19 & 4.24 & \\
\hline $7-3,90$ & 59.00 & 1.91 & 0.26 & 0.22 & 14.06 & \\
\hline $7-3,100$ & 59.10 & 2.64 & 0.27 & 0.22 & 20.21 & \\
\hline $7-3,120$ & 59.30 & 3.20 & 0.21 & 0.16 & 25.36 & \\
\hline $7-3,140$ & 59.50 & 1.86 & 0.21 & 0.18 & 14.00 & \\
\hline $7-4,9$ & 59.69 & 2.17 & 0.24 & 0.20 & 16.41 & \\
\hline $7-4,30$ & 59.90 & 1.88 & 0.35 & 0.30 & 13.13 & \\
\hline $7-4,49$ & 60.09 & 0.80 & 0.29 & 0.28 & 4.36 & \\
\hline $7-4,70$ & 60.30 & 0.61 & 0.23 & 0.22 & 3.23 & \\
\hline $7-4,90$ & 60.50 & 0.54 & 0.18 & 0.17 & 3.05 & \\
\hline $7-4,110$ & 60.60 & 0.68 & 0.15 & 0.14 & 4.47 & \\
\hline $7-4,130$ & 60.90 & 1.16 & 0.14 & 0.13 & 8.60 & \\
\hline $7-4,149$ & 61.09 & 1.30 & 0.18 & 0.16 & 9.48 & \\
\hline $7-5,23$ & 61.33 & 1.02 & 0.24 & 0.22 & 6.63 & \\
\hline $7-5,40$ & 61.50 & 1.05 & 0.21 & 0.20 & 7.13 & \\
\hline $7-5,60$ & 61.70 & 0.30 & 0.09 & 0.09 & 1.76 & \\
\hline $7-5,80$ & 61.90 & 1.85 & 0.14 & 0.12 & 14.42 & \\
\hline $7-5,100$ & 62.10 & 3.46 & 0.29 & 0.21 & 27.07 & \\
\hline $7-5,119$ & 62.29 & 1.77 & 0.25 & 0.22 & 12.94 & \\
\hline $7-5,139$ & 62.49 & 3.18 & 0.23 & 0.17 & 25.07 & \\
\hline $7-6,9$ & 62.69 & 1.25 & 0.22 & 0.20 & 8.74 & \\
\hline $7-6,30$ & 62.90 & 1.12 & 0.16 & 0.15 & 8.11 & \\
\hline $7-6,50$ & 63.10 & 0.59 & 0.18 & 0.17 & 3.47 & \\
\hline $7-6,70$ & 63.30 & 1.25 & 0.23 & 0.21 & 8.67 & \\
\hline $7-6,90$ & 63.50 & 2.83 & 0.18 & 0.14 & 22.42 & \\
\hline $7-6,110$ & 63.70 & 2.39 & 0.18 & 0.15 & 18.70 & \\
\hline $7-6,130$ & 63.90 & 1.15 & 0.20 & 0.18 & 8.05 & \\
\hline
\end{tabular}


Table 4 (continued).

\begin{tabular}{|c|c|c|c|c|c|c|}
\hline $\begin{array}{l}\text { Core/section } \\
\text { interval }(\mathrm{cm})\end{array}$ & $\begin{array}{l}\text { Depth } \\
\text { (mbsf) }\end{array}$ & $\mathrm{C}_{\text {tot }}$ & $\mathrm{C}_{\text {org' }}$ & $\mathrm{C}_{\text {org }}$ & $\mathrm{CaCO}_{3}$ & $\mathrm{C} / \mathrm{N}$ \\
\hline \multicolumn{7}{|l|}{ D } \\
\hline $105-646 \mathrm{~B}-21-1,30$ & 188.50 & 0.42 & 0.41 & 0.41 & 0.09 & \\
\hline $21-1,60$ & 188.80 & 0.48 & 0.48 & 0.48 & 0.00 & \\
\hline $21-1,74$ & 188.94 & 0.40 & 0.40 & 0.40 & 0.00 & \\
\hline $21-1,104$ & 189.24 & 0.35 & 0.31 & 0.31 & 0.34 & \\
\hline $21-1,120$ & 189.40 & 0.32 & 0.32 & 0.32 & 0.00 & \\
\hline $21-1,140$ & 189.60 & 0.27 & 0.27 & 0.27 & 0.00 & \\
\hline $21-2,10$ & 189.80 & 0.27 & 0.27 & 0.27 & 0.00 & \\
\hline $21-2,30$ & 190.00 & 0.33 & 0.33 & 0.33 & 0.00 & \\
\hline $21-2,50$ & 190.20 & 0.37 & 0.34 & 0.34 & 0.26 & \\
\hline $21-2,72$ & 190.42 & 0.40 & 0.35 & 0.35 & 0.43 & \\
\hline $21-2,90$ & 190.60 & 1.20 & 0.22 & 0.20 & 8.32 & \\
\hline $21-2,110$ & 190.80 & 0.47 & 0.19 & 0.19 & 2.37 & \\
\hline $21-2,129$ & 190.99 & 0.52 & 0.29 & 0.28 & 1.96 & \\
\hline $21-2,149$ & 191.19 & 0.35 & 0.35 & 0.35 & 0.00 & \\
\hline $21-3,16$ & 191.36 & 0.30 & 0.30 & 0.30 & 0.00 & \\
\hline $21-3,33$ & 191.53 & 0.32 & 0.31 & 0.31 & 0.09 & \\
\hline $21-3,50$ & 191.70 & 0.53 & 0.53 & 0.53 & 0.00 & \\
\hline $21-3,70$ & 191.90 & 0.43 & 0.38 & 0.38 & 0.43 & \\
\hline $21-3,90$ & 192.10 & 0.40 & 0.32 & 0.32 & 0.68 & \\
\hline $21-3,110$ & 192.30 & 0.74 & 0.28 & 0.27 & 3.93 & \\
\hline $21-3,131$ & 192.51 & 0.31 & 0.22 & 0.22 & 0.76 & \\
\hline $21-4,1$ & 192.71 & 0.51 & 0.46 & 0.46 & 0.43 & \\
\hline $21-4,17$ & 192.87 & 0.63 & 0.63 & 0.63 & 0.00 & \\
\hline $21-4,32$ & 193.02 & 0.54 & 0.54 & 0.54 & 0.00 & \\
\hline $21-4,50$ & 193.20 & 0.53 & 0.53 & 0.53 & 0.00 & \\
\hline $21-4,71$ & 193.41 & 0.36 & 0.36 & 0.36 & 0.00 & \\
\hline $21-4,84$ & 193.54 & 0.66 & 0.61 & 0.61 & 0.44 & \\
\hline $21-4,105$ & 193.75 & 0.33 & 0.33 & 0.33 & 0.00 & \\
\hline $21-4,125$ & 193.95 & 0.29 & 0.29 & 0.29 & 0.00 & \\
\hline $21-5,0$ & 194.20 & 0.29 & 0.27 & 0.27 & 0.17 & \\
\hline $21-5,24$ & 194.44 & 0.25 & 0.25 & 0.25 & 0.00 & \\
\hline \multicolumn{7}{|l|}{$\mathrm{E}$} \\
\hline $105-646 \mathrm{~B}-37-1,22$ & 343.02 & 0.86 & 0.29 & 0.28 & 4.87 & \\
\hline $37-1,40$ & 343.20 & 0.94 & 0.34 & 0.32 & 5.15 & \\
\hline $37-1,59$ & 343.39 & 0.58 & 0.25 & 0.24 & 2.81 & \\
\hline $37-1,80$ & 343.60 & 0.71 & 0.32 & 0.31 & 3.34 & \\
\hline $37-1,102$ & 343.82 & 0.68 & 0.36 & 0.35 & 2.75 & \\
\hline $37-1,120$ & 344.00 & 0.56 & 0.35 & 0.34 & 1.80 & \\
\hline $37-1,145$ & 344.25 & 0.56 & 0.38 & 0.37 & 1.55 & \\
\hline $37-2,20$ & 344.50 & 0.46 & 0.32 & 0.32 & 1.20 & \\
\hline $37-2,40$ & 344.70 & 0.45 & 0.29 & 0.29 & 1.37 & \\
\hline $37-2,55$ & 344.85 & 0.70 & 0.30 & 0.29 & 3.42 & \\
\hline $37-2,72$ & 345.02 & 2.04 & 0.27 & 0.23 & 15.09 & \\
\hline $37-2,90$ & 345.20 & 1.85 & 0.33 & 0.29 & 13.03 & \\
\hline $37-2,111$ & 345.41 & 0.81 & 0.32 & 0.31 & 4.20 & \\
\hline $37-2,132$ & 345.68 & 0.46 & 0.32 & 0.32 & 1.20 & \\
\hline $37-3,5$ & 345.85 & 2.32 & 0.32 & 0.27 & 17.12 & \\
\hline $37-3,25$ & 346.05 & 3.07 & 0.35 & 0.27 & 23.35 & \\
\hline $37-3,45$ & 346.25 & 3.55 & 0.34 & 0.25 & 27.53 & \\
\hline $37-3,65$ & 346.45 & 2.92 & 0.32 & 0.25 & 22.26 & \\
\hline $37-3,88$ & 346.68 & 1.92 & 0.38 & 0.33 & 13.25 & \\
\hline $37-3,110$ & 346.90 & 2.06 & 0.35 & 0.30 & 14.68 & \\
\hline $37-3,128$ & 347.08 & 1.55 & 0.39 & 0.35 & 9.99 & \\
\hline $37-3,148$ & 347,28 & 0.88 & 0.38 & 0.36 & 4.30 & \\
\hline $37-4,20$ & 347.50 & 1.19 & 0.40 & 0.37 & 6.81 & \\
\hline $37-4,40$ & 347.70 & 0.71 & 0.34 & 0.33 & 3.17 & \\
\hline $37-4,58$ & 347.88 & 0.65 & 0.30 & 0.29 & 2.99 & \\
\hline $37-4,79$ & 348.09 & 0.71 & 0.27 & 0.26 & 3.75 & \\
\hline $37-4,100$ & 348.30 & 0.70 & 0.27 & 0.26 & 3.67 & \\
\hline $37-4,120$ & 348.50 & 1.91 & 0.30 & 0.26 & 13.76 & \\
\hline $37-4,141$ & 348.71 & 3.14 & 0.28 & 0.21 & 24.40 & \\
\hline $37-5,12$ & 348.92 & 1.32 & 0.40 & 0.37 & 7.93 & \\
\hline $37-5,28$ & 349.08 & 1.41 & 0.36 & 0.33 & 9.02 & \\
\hline $37-5,53$ & 349.33 & 0.79 & 0.34 & 0.33 & 3.86 & \\
\hline $37-5,70$ & 349.50 & 0.75 & 0.30 & 0.29 & 3.85 & \\
\hline $37-5,93$ & 349.73 & 0.73 & 0.35 & 0.34 & 3.26 & \\
\hline $37-5,110$ & 349.90 & 0.70 & 0.32 & 0.31 & 3.25 & \\
\hline $37-5,134$ & 350.14 & 0.48 & 0.28 & 0.28 & 1.71 & \\
\hline $37-6,5$ & 350.35 & 0.47 & 0.28 & 0.28 & 1.58 & \\
\hline
\end{tabular}

$\mathrm{C}_{\text {tot }}=$ total carbon; $\mathrm{C}_{\mathrm{org}^{\prime}}=$ total organic carbon as a percentage of the carbonate-free sample; $\mathrm{C}_{\text {org }}=$ total organic carbon as a percentage of the whole sample; $\mathrm{CaCO}_{3}=$ carbonate contents; $\mathrm{C} / \mathrm{N}=$ total organic carbon/total nitrogen; $\mathrm{A}=$ long-term record; $\mathrm{B}-\mathrm{E}=$ shortterm records. 
Table 5. Total carbon, total organic carbon, carbonate contents, and $\mathrm{C} / \mathrm{N}$ ratios at Site 647 .

\begin{tabular}{|c|c|c|c|c|c|c|}
\hline $\begin{array}{l}\text { Core/section } \\
\text { interval }(\mathrm{cm})\end{array}$ & $\begin{array}{l}\text { Depth } \\
\text { (mbsf) }\end{array}$ & $C_{\text {tot }}$ & $\mathrm{C}_{\text {org }}$ & $\mathrm{CaCO}_{3}$ & $\mathrm{C}_{\text {org }}$ & $\mathrm{C} / \mathrm{N}$ \\
\hline $105-647 \mathrm{~A}-1-1,69$ & 0.69 & 2.35 & 0.27 & 17.7 & 0.22 & 7 \\
\hline $1-1,75$ & 0.75 & & & 21.8 & 0.15 & \\
\hline $1-2,60$ & 2.10 & & & 8.0 & & \\
\hline $1-6,75$ & 8.25 & & & 19.0 & & \\
\hline $2-1,85$ & 10.05 & & & 12.7 & 0.20 & \\
\hline $2-1,129$ & 10.39 & 5.50 & & & & \\
\hline $2-6,55$ & 17.25 & 1.78 & & 10.0 & & \\
\hline $4-1,135$ & 29.95 & & & 8.1 & 0.26 & \\
\hline $4-3,65$ & 32.25 & & & 16.0 & & \\
\hline $4-4,106$ & 34.16 & 1.81 & 0.32 & 12.8 & 0.28 & 8 \\
\hline $4-4,128$ & 34.38 & 3.95 & 0.22 & 31.7 & 0.15 & 7 \\
\hline $4-5,75$ & 35.35 & 3.11 & & 26.2 & 0.19 & \\
\hline $6-1,70$ & 49.30 & & & 16.6 & 0.52 & \\
\hline $6-3,75$ & 52.35 & & & 32.0 & & \\
\hline $6-3,118$ & 52.78 & 3.03 & 0.22 & 23.9 & 0.17 & 6 \\
\hline $6-5,70$ & 55.30 & 1.24 & & 38.3 & 0.14 & \\
\hline $6-5,124$ & 55.84 & 1.43 & 0.34 & 9.3 & 0.31 & 9 \\
\hline $6-6,18$ & 56.28 & 1.65 & 0.34 & 11.2 & 0.30 & 9 \\
\hline $7-2,72$ & 60.52 & & & 29.0 & & \\
\hline $7-4,75$ & 63.55 & & & 22.0 & & \\
\hline $7-5,34$ & 64.64 & 2.72 & 0.25 & 21.0 & 0.20 & 6 \\
\hline $7-5,119$ & 65.49 & & & 21.7 & 0.13 & \\
\hline $7-6,75$ & 66.55 & & & 20.0 & & \\
\hline $8-1,74$ & 68.74 & & & 23.0 & & \\
\hline $8-3,65$ & 71.65 & & & 12.0 & 0.13 & \\
\hline $8-3,46$ & 71.66 & 2.72 & 0.30 & 20.7 & 0.24 & 5 \\
\hline $9-1,77$ & 78.37 & 1.05 & & 7.0 & 0.21 & \\
\hline $9-2,80$ & 79.90 & & & 13.7 & 0.36 & \\
\hline $9-3,69$ & 81.29 & 1.55 & 0.58 & 8.5 & 0.53 & 15 \\
\hline $10-1,75$ & 88.05 & & & 5.0 & & \\
\hline $10-2,77$ & 89.57 & 3.90 & 0.29 & 30.8 & 0.20 & 10 \\
\hline $10-3,77$ & 91.07 & & & 8.0 & & \\
\hline $10-3,119$ & 91.49 & & & 17.8 & 0.32 & \\
\hline $10-5,40$ & 93.70 & & & 7.0 & & \\
\hline $11-1,80$ & 97.80 & & & 15.0 & & \\
\hline $11-3,56$ & 100.56 & 0.85 & 0.45 & 3.5 & 0.43 & 11 \\
\hline $11-3,80$ & 100.80 & 0.83 & & 3.5 & 0.43 & \\
\hline $12-1,70$ & 107.40 & & & 5.8 & 0.34 & \\
\hline $12-2,77$ & 108.99 & 0.60 & 0.51 & 0.8 & 0.51 & 13 \\
\hline $12-3,79$ & 110.49 & 0.55 & & 5.0 & & \\
\hline $13-1,64$ & 116.64 & & & 11.0 & & \\
\hline $13-1,75$ & 116.75 & 1.45 & & 10.0 & 0.25 & \\
\hline $13-3,29$ & 119.29 & 0.10 & 0.10 & 0.0 & 0.10 & 3 \\
\hline $13-3,76$ & 119.76 & & & 0.0 & & \\
\hline $13-4,119$ & 121.69 & 0.11 & & 0.1 & 0.14 & \\
\hline $13-5,110$ & 123.10 & 0.14 & & 0.0 & 0.14 & \\
\hline $14-1,20$ & 125.90 & 0.12 & 0.10 & 0.2 & 0.10 & 3 \\
\hline $14-1,102$ & 126.72 & 0.16 & & 0.0 & & \\
\hline $14-3,37$ & 129.07 & 0.17 & & 0.0 & 0.10 & \\
\hline $15-1,57$ & 135.97 & 1.57 & 0.37 & 10.3 & 0.33 & 6 \\
\hline $15-1,109$ & 136.49 & 1.31 & & 7.0 & 0.47 & \\
\hline $15-3,70$ & 139.10 & 2.86 & & 19.4 & 0.32 & \\
\hline $16-1,98$ & 146.08 & 2.05 & & 9.0 & & \\
\hline $16-3,02$ & 148.12 & 1.40 & 0.38 & 8.8 & 0.35 & 6 \\
\hline $16-3,14$ & 148.24 & 4.14 & 0.38 & 32.4 & 0.26 & 5 \\
\hline $16-3,31$ & 148.41 & 4.65 & & 35.0 & 0.45 & \\
\hline $17-1,19$ & 154.99 & & & 33.0 & & \\
\hline $17-1,55$ & 155.35 & 3.55 & 0.44 & 26.9 & 0.32 & 7 \\
\hline $17-1,65$ & 155.45 & 3.54 & 0.28 & 27.8 & 0.20 & 6 \\
\hline $17-3,101$ & 158.81 & 5.21 & & 42.0 & 0.17 & \\
\hline $17-4,107$ & 160.37 & 3.75 & 0.36 & 29.1 & 0.26 & 7 \\
\hline $17-4,119$ & 160.49 & & & 34.5 & 0.23 & \\
\hline $17-5,129$ & 162.09 & & & 32.0 & & \\
\hline $18-1,93$ & 165.93 & 4.01 & 0.45 & 30.8 & 0.31 & 6 \\
\hline $18-2,93$ & 166.53 & 6.12 & & 48.9 & 0.17 & \\
\hline $19-1,84$ & 174.64 & 2.63 & & 40.0 & & \\
\hline $19-3,51$ & 177.31 & 5.55 & 0.48 & 44.0 & 0.27 & 8 \\
\hline $19-3,88$ & 177.68 & 5.24 & & 44.7 & 0.27 & \\
\hline $19-5,100$ & 179.80 & 3.44 & 0.28 & 27.0 & 0.20 & 5 \\
\hline $19-5,121$ & 181.01 & 4.52 & & 38.0 & & \\
\hline $20-1,50$ & 184.00 & 4.09 & & 39.0 & & \\
\hline $20-3,43$ & 186.93 & 3.23 & 0.47 & 23.9 & 0.36 & 6 \\
\hline $20-3,48$ & 186.98 & 2.51 & & 20.0 & 0.11 & \\
\hline $20-4,119$ & 189.19 & & & 18.3 & 0.34 & \\
\hline $20-5,75$ & 190.25 & & & 27.0 & & \\
\hline $21-1,26$ & 193.35 & & & 22.4 & & \\
\hline $21-1,90$ & 194.00 & 2.48 & 0.42 & 17.8 & 0.35 & 7 \\
\hline
\end{tabular}

Table 5 (continued).

\begin{tabular}{|c|c|c|c|c|c|c|}
\hline $\begin{array}{l}\text { Core/section } \\
\text { interval }(\mathrm{cm})\end{array}$ & $\begin{array}{l}\text { Depth } \\
\text { (mbsf) }\end{array}$ & $\mathrm{C}_{\text {tot }}$ & $\mathrm{C}_{\text {org }}{ }^{\prime}$ & $\mathrm{CaCO}_{3}$ & $\mathrm{C}_{\text {org }}$ & $\mathrm{C} / \mathrm{N}$ \\
\hline $105-647 \mathrm{~A}-21-1,100$ & 194.10 & & & 16.0 & 0.34 & \\
\hline $21-3,110$ & 197.20 & 1.60 & & 40.0 & & \\
\hline $22-1,10$ & 202.80 & & & 7.8 & 0.48 & \\
\hline $23-1,19$ & 212.59 & & & 0.6 & & \\
\hline $23-1,114$ & 213.44 & 0.54 & 0.50 & 0.3 & 0.50 & \\
\hline $23-2,37$ & 214.17 & 0.50 & 0.50 & 0.0 & 0.50 & 8 \\
\hline $23-3,91$ & 216.21 & 1.41 & & 12.0 & & \\
\hline $23-4,118$ & 217.98 & & & 2.5 & & \\
\hline $23-5,49$ & 218.79 & 0.88 & & 5.0 & 0.28 & \\
\hline $24-1,51$ & 222.41 & & & 4.6 & & \\
\hline $24-1,130$ & 223.20 & 0.69 & & 6.0 & & \\
\hline $24-3,10$ & 225.00 & 0.89 & & 4.0 & 0.41 & \\
\hline $24-3,101$ & 225.91 & 2.11 & 0.56 & 13.6 & 0.48 & 11 \\
\hline $24-3,119$ & 226.09 & 1.20 & & 7.3 & 0.40 & \\
\hline $25-1,56$ & 232.06 & & & 12.1 & & \\
\hline $25-3,123$ & 235.73 & & & 12.8 & 0.37 & \\
\hline $25-3,136$ & 235.86 & 1.94 & 0.60 & 11.8 & 0.53 & 10 \\
\hline $25-4,95$ & 236.95 & & & 6.0 & & \\
\hline $25-5,145$ & 238.95 & & & 17.9 & 0.37 & \\
\hline $27-1,101$ & 251.41 & & & 33.8 & 0.27 & \\
\hline $28-1,95$ & 261.05 & & & 30.0 & & \\
\hline $28-3,80$ & 263.90 & & & 43.0 & & \\
\hline $28-4,20$ & 264.82 & 2.09 & 0.38 & 14.7 & 0.32 & 8 \\
\hline $28-5,119$ & 267.29 & & & 28.2 & 0.30 & \\
\hline $30-1,75$ & 280.15 & & & 45.0 & & \\
\hline $30-3,32$ & 282.72 & 5.87 & 0.28 & 47.7 & 0.15 & 6 \\
\hline $30-3,73$ & 283.13 & & & 45.8 & 0.17 & \\
\hline $30-5,75$ & 286.15 & & & 36.0 & 0.15 & \\
\hline $31-2,75$ & 291.25 & & & & & \\
\hline $32-1,22$ & 298.82 & & & 33.5 & 0.26 & \\
\hline $32-1,26$ & 298.86 & 4.28 & & 36.0 & & \\
\hline $32-2,38$ & 300.48 & & & 33.6 & 0.17 & \\
\hline $33-1,96$ & 309.26 & 4.77 & 37.9 & 0.22 & & \\
\hline $33-2,47$ & 310.27 & & & 35.5 & 0.18 & \\
\hline $34-1,50$ & 318.50 & & & 27.1 & 0.18 & \\
\hline $35-1,93$ & 328.53 & & & 29.0 & & \\
\hline $35-2,119$ & 330.29 & & & 30.8 & 0.21 & \\
\hline $35-3,103$ & 331.63 & & & 30.0 & & \\
\hline $36-1,118$ & 338.38 & & & 24.0 & & \\
\hline $36-3,77$ & 340.97 & 3.41 & & 25.8 & 0.32 & \\
\hline $36-3,83$ & 341.03 & & & 28.0 & & \\
\hline $36-3,100$ & 341.21 & & & 31.0 & & \\
\hline $36-4,105$ & 342.75 & & & 26.9 & 0.23 & \\
\hline $37-1,110$ & 347.80 & & & 28.0 & & \\
\hline $37-2,97$ & 349.17 & 4.58 & & 36.3 & 0.22 & \\
\hline $37-3,79$ & 350.49 & & & 29.8 & 0.22 & \\
\hline $38-1,23$ & 356.63 & 4.94 & & 39.1 & 0.25 & \\
\hline $38-1,94$ & 357.34 & & & 32.0 & & \\
\hline $38-3,44$ & 359.84 & & & 33.0 & & \\
\hline $38-5,108$ & 363.48 & & & 25.0 & & \\
\hline $38-5,119$ & 363.59 & & & 21.0 & 0.23 & \\
\hline $39-1,56$ & 366.66 & 2.88 & & 21.4 & 0.32 & \\
\hline $39-1,99$ & 367.09 & & & 24.2 & 0.26 & \\
\hline $39-3,25$ & 369.35 & & & 31.0 & & \\
\hline $41-1,126$ & 386.66 & & & 38.0 & & \\
\hline $41-3,116$ & 389.56 & & & 37.0 & & \\
\hline $41-4,80$ & 390.70 & 5.16 & & 41.5 & 0.18 & \\
\hline $41-5,110$ & 392.50 & & & 42.0 & & \\
\hline $41-5,119$ & 392.59 & & & 40.2 & 0.15 & \\
\hline $41-6,128$ & 394.18 & & & 47.0 & & \\
\hline $42-1,57$ & 395.67 & & & 32.0 & & \\
\hline $42-1,64$ & 395.74 & 3.58 & & 28.5 & 0.16 & \\
\hline $42-1,75$ & 395.85 & & & 36.0 & & \\
\hline $42-2,69$ & 397.29 & 5.97 & & 48.5 & 0.16 & \\
\hline $42-3,85$ & 398.95 & & & 41.9 & 0.15 & \\
\hline $42-4,113$ & 400.73 & & & 49.0 & & \\
\hline $42-5,76$ & 401.86 & & & 50.0 & & \\
\hline $43-1,62$ & 405.42 & & & 40.0 & & \\
\hline $43-2,71$ & 407.01 & & & 36.0 & & \\
\hline $43-2,85$ & 407.15 & 2.54 & & 19.2 & 0.24 & \\
\hline $43-5,65$ & 411.75 & & & 36.4 & 0.21 & \\
\hline $44-1,57$ & 415.07 & & & 2.6 & 0.26 & \\
\hline $44-2,25$ & 416.25 & & & 36.0 & & \\
\hline $44-2,50$ & 416.50 & & & 38.0 & & \\
\hline $44-3,09$ & 417.49 & 3.53 & & 28.1 & 0.16 & \\
\hline $44-3,136$ & 418.86 & 5.56 & & 45.3 & 0.13 & \\
\hline $44-4,50$ & 419.50 & & & 54.0 & & \\
\hline $44-4,82$ & 419.82 & 7.18 & & 59.0 & 0.10 & \\
\hline
\end{tabular}


Table 5 (continued).

\begin{tabular}{|c|c|c|c|c|c|c|}
\hline $\begin{array}{l}\text { Core/section } \\
\text { interval }(\mathrm{cm})\end{array}$ & $\begin{array}{l}\text { Depth } \\
\text { (mbsf) }\end{array}$ & $C_{\text {tot }}$ & $\mathrm{C}_{\text {org }^{\prime}}$ & $\mathrm{CaCO}_{3}$ & $\mathrm{C}_{\text {org }}$ & $\mathrm{C} / \mathrm{N}$ \\
\hline $105-647 \mathrm{~A}-44-5,75$ & 421.25 & & & 52.0 & & \\
\hline $44-5,119$ & 421.69 & & & 47.6 & 0.15 & \\
\hline $45-2,10$ & 425.70 & & & 45.0 & & \\
\hline $45-2,47$ & 426.07 & 4.49 & & 35.0 & 0.19 & \\
\hline $45-3,26$ & 427.36 & & & 35.0 & & \\
\hline $45-4,150$ & 430.10 & & & 33.6 & 0.20 & \\
\hline $46-2,74$ & 436.04 & & & 29.0 & & \\
\hline $46-3,68$ & 437.48 & 1.93 & & 14.5 & 0.19 & \\
\hline $46-5,70$ & 440.50 & & & 21.8 & & \\
\hline $47-1,44$ & 443.94 & & & 16.0 & & \\
\hline $47-3,53$ & 447.03 & 2.68 & & 20.8 & 0.19 & \\
\hline $47-4,121$ & 449.21 & & & 38.0 & & \\
\hline $47-4,141$ & 449.41 & & & 45.0 & & \\
\hline $47-5,119$ & 450.69 & & & 0.3 & 0.18 & \\
\hline $48-2,51$ & 455.21 & & & 18.0 & & \\
\hline $48-3,127$ & 457.47 & 3.07 & & 22.9 & 0.33 & \\
\hline $48-5,71$ & 459.91 & & & 19.3 & 0.26 & \\
\hline $49-1,59$ & 463.37 & 3.03 & & 21.5 & 0.45 & \\
\hline $49-2,97$ & 465.27 & & & 20.8 & 0.17 & \\
\hline $49-4,96$ & 468.26 & & & 38.0 & & \\
\hline $49-5,113$ & 469.93 & 4.69 & & 37.0 & 0.25 & \\
\hline $50-2,88$ & 474.88 & & & 29.0 & & \\
\hline $50-4,82$ & 477.82 & 3.41 & & 26.9 & 0.19 & \\
\hline $50-5,58$ & 479.08 & & & 35.0 & & \\
\hline $50-6,119$ & 481.19 & & & 33.8 & 0.17 & \\
\hline $51-2,32$ & 483.92 & & & 39.0 & & \\
\hline $51-4,82$ & 487.42 & 2.22 & & 16.4 & 0.25 & \\
\hline $51-6,64$ & 490.24 & & & 36.6 & 0.18 & \\
\hline $52-3,59$ & 495.39 & & & 29.5 & 0.20 & \\
\hline $52-4,102$ & 497.32 & 5.06 & & 40.0 & 0.23 & \\
\hline $52-6,91$ & 500.21 & & & 29.0 & & \\
\hline $53-2,69$ & 503.59 & & & 32.0 & & \\
\hline $53-5,64$ & 508.04 & & & 36.0 & & \\
\hline $53-5,119$ & 508.59 & & & 38.1 & 0.10 & \\
\hline $54-1,45$ & 511.55 & 3.57 & & 27.5 & 0.27 & \\
\hline $54-1,136$ & 512.46 & & & 26.8 & 0.20 & \\
\hline $54-5,114$ & 518.24 & & & 31.5 & 0.17 & \\
\hline $54-6,02$ & 518.62 & & & 35.0 & & \\
\hline $62-2,114$ & 600.74 & & & 24.6 & 0.05 & \\
\hline $62-4,73$ & 603.33 & & & 38.0 & & \\
\hline $62-5,119$ & 605.29 & 3.54 & & 27.4 & 0.25 & \\
\hline $62-6,31$ & 605.91 & & & 33.0 & & \\
\hline $63-1,128$ & 609.08 & & & 28.7 & 0.13 & \\
\hline $64-1,24$ & 617.74 & & & 3.0 & & \\
\hline $64-1,49$ & 617.99 & 0.26 & & 0.8 & 0.16 & \\
\hline $64-1,95$ & 618.45 & & & 0.0 & 0.20 & \\
\hline $64-3,59$ & 621.09 & 0.14 & & & & \\
\hline $64-3,106$ & 621.56 & & & 0.0 & 0.25 & \\
\hline $64-3,110$ & 621.60 & & & 0.0 & & \\
\hline $65-1,28$ & 627.48 & 0.35 & & 0.0 & 0.35 & \\
\hline $65-1,56$ & 627.76 & & & 3.0 & & \\
\hline $65-2,119$ & 629.89 & & & 0.0 & 0.27 & \\
\hline $65-2,134$ & 630.04 & 0.29 & & & & \\
\hline $65-3,135$ & 631.55 & & & 4.0 & & \\
\hline $66-1,94$ & 637.74 & & & 11.7 & 0.10 & \\
\hline $66-3,41$ & 640.21 & 1.38 & & 10.5 & 0.14 & \\
\hline $66-3,77$ & 640.57 & & & 10.0 & & \\
\hline $67-1,27$ & 646.77 & & & 12.5 & 0.07 & \\
\hline $67-2,82$ & 648.82 & 1.10 & & & & \\
\hline $67-4,02$ & 651.02 & & & 8.0 & & \\
\hline $68-1,31$ & 656.51 & & & 18.5 & 0.06 & \\
\hline $68-1,52$ & 656.72 & & & 13.0 & & \\
\hline $68-2,111$ & 658.81 & & & 12.0 & & \\
\hline $68-4,05$ & 660.75 & 1.49 & & 12.4 & 0.00 & \\
\hline $68-4,113$ & 661.83 & & & & & \\
\hline $70-1,71$ & 685.91 & & & 26.5 & 0.12 & \\
\hline $70-2,133$ & 688.03 & & & & & \\
\hline $70-3,119$ & 689.39 & & & 27.2 & 0.04 & \\
\hline $70-4,39$ & 690.09 & & & 25.0 & & \\
\hline $71-1,27$ & 695.07 & 3.38 & & 26.9 & 0.15 & \\
\hline $71-1,116$ & 695.96 & & & 27.2 & 0.08 & \\
\hline
\end{tabular}

$\mathrm{C}_{\mathrm{tot}}=$ total carbon; $\mathrm{C}_{\mathrm{org}^{\prime}}=$ total organic carbon as a percentage of the carbonate-free sample; $\mathrm{C}_{\mathrm{org}}=$ total organic carbon as a percentage of the whole sample; $\mathrm{CaCO}_{3}=$ carbonate contents; $\mathrm{C} / \mathrm{N}=$ total carbon/total nitrogen ratio. 
R. STEIN, R. LITTKE, R. STAX, D. WELTE

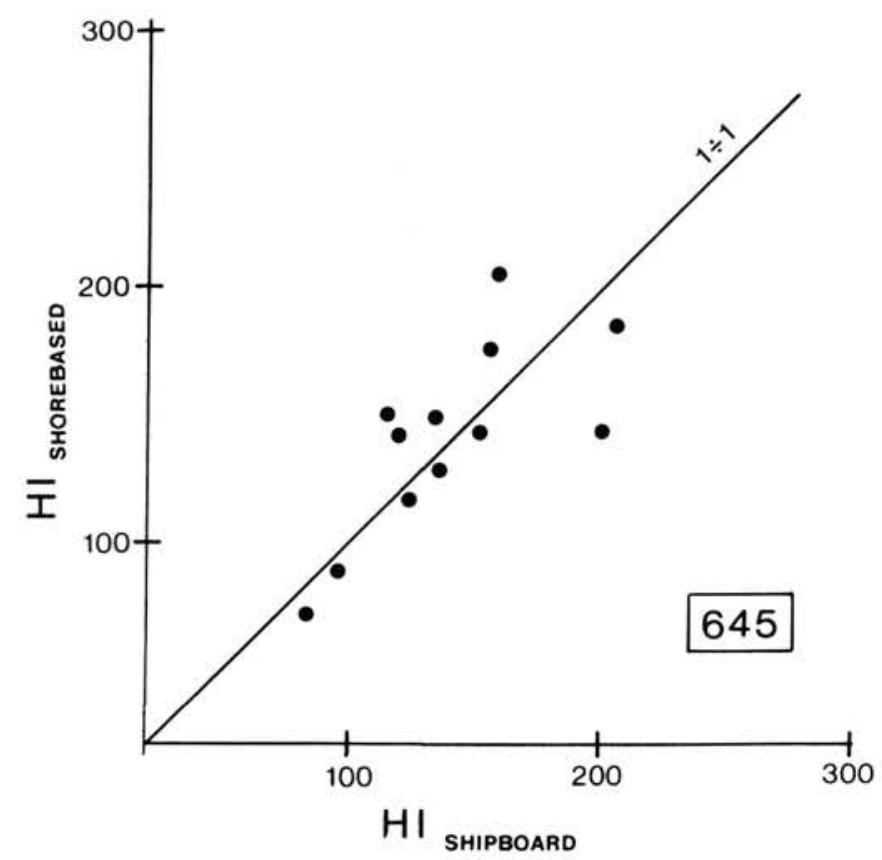

Figure 4. Comparison of hydrogen index values determined by RockEval pyrolysis on board JOIDES Resolution and in our laboratory.

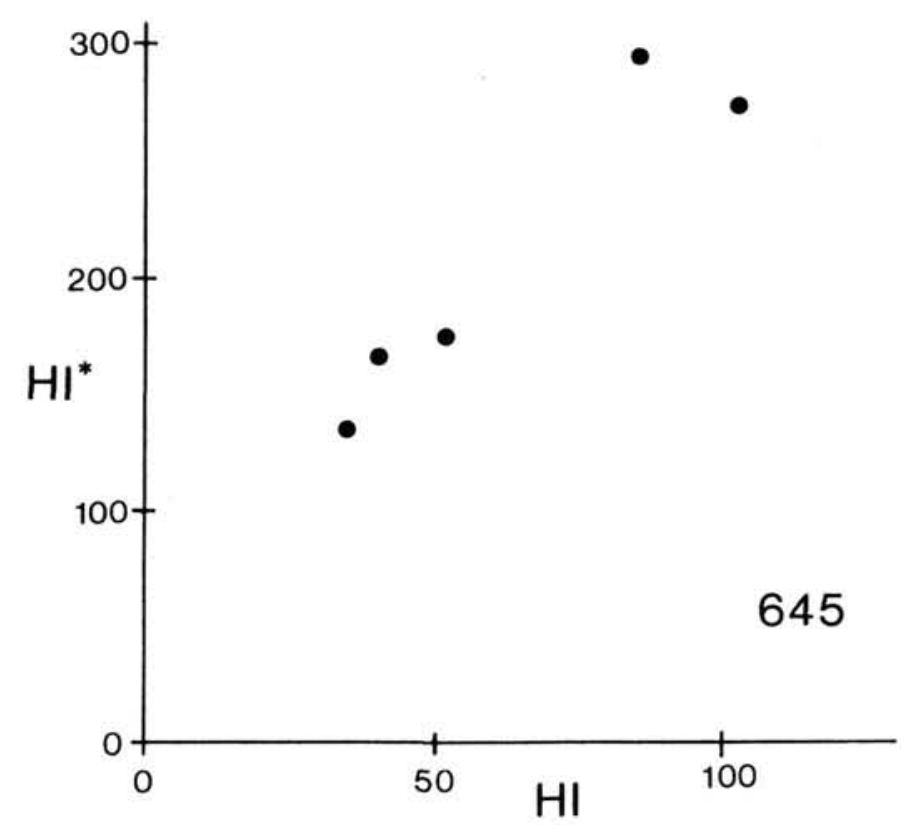

Figure 5. Comparison of hydrogen index values measured on bulk-sediment samples (HI) and kerogen concentrates ( $\left.\mathrm{HI}^{*}\right)$. 


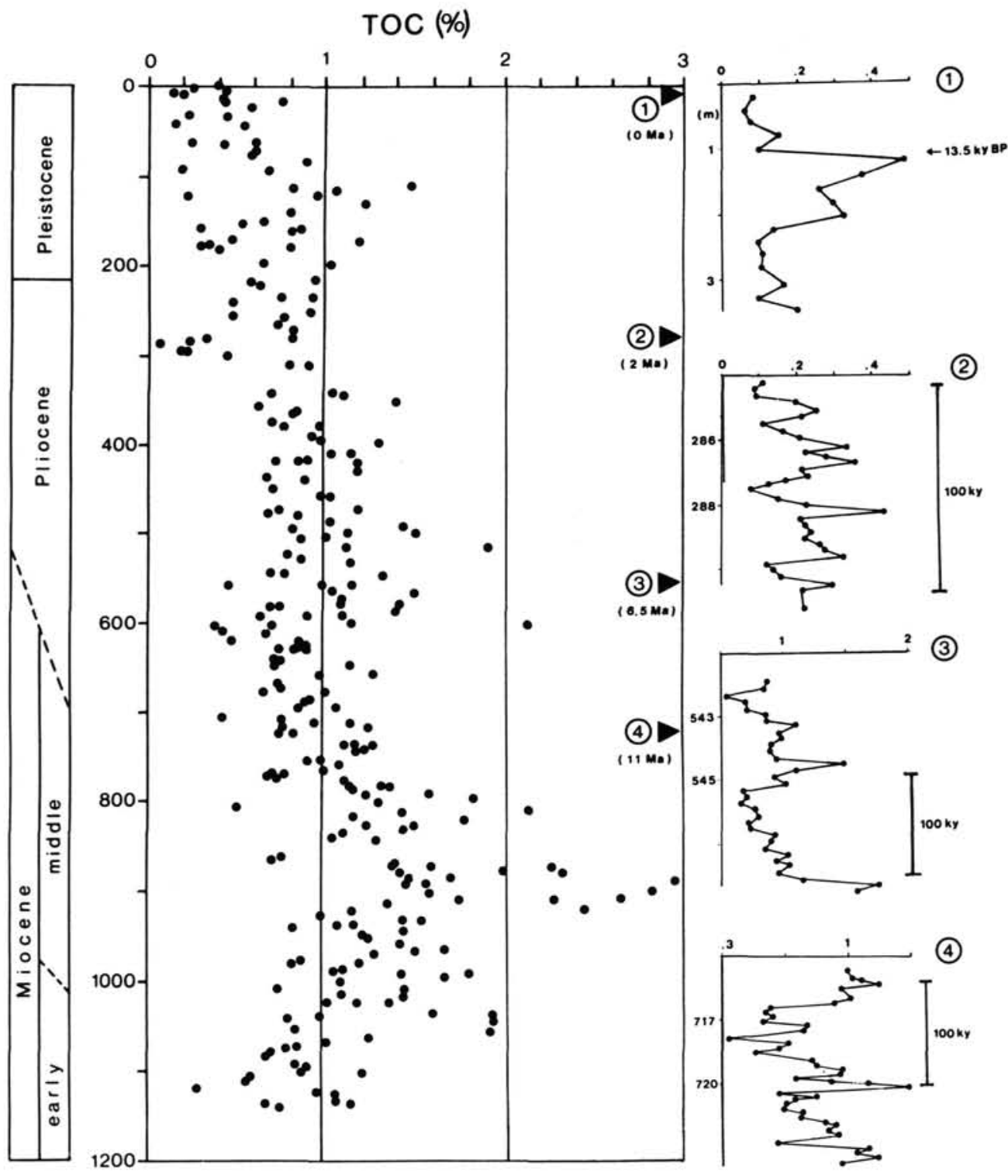

Figure 6. Total-organic-carbon contents at Site 645. The long-term record includes shipboard data and data from Tables 1 and 3; the four short-term records in the right show data from Table 2. 


\section{6}

TOC (\%)
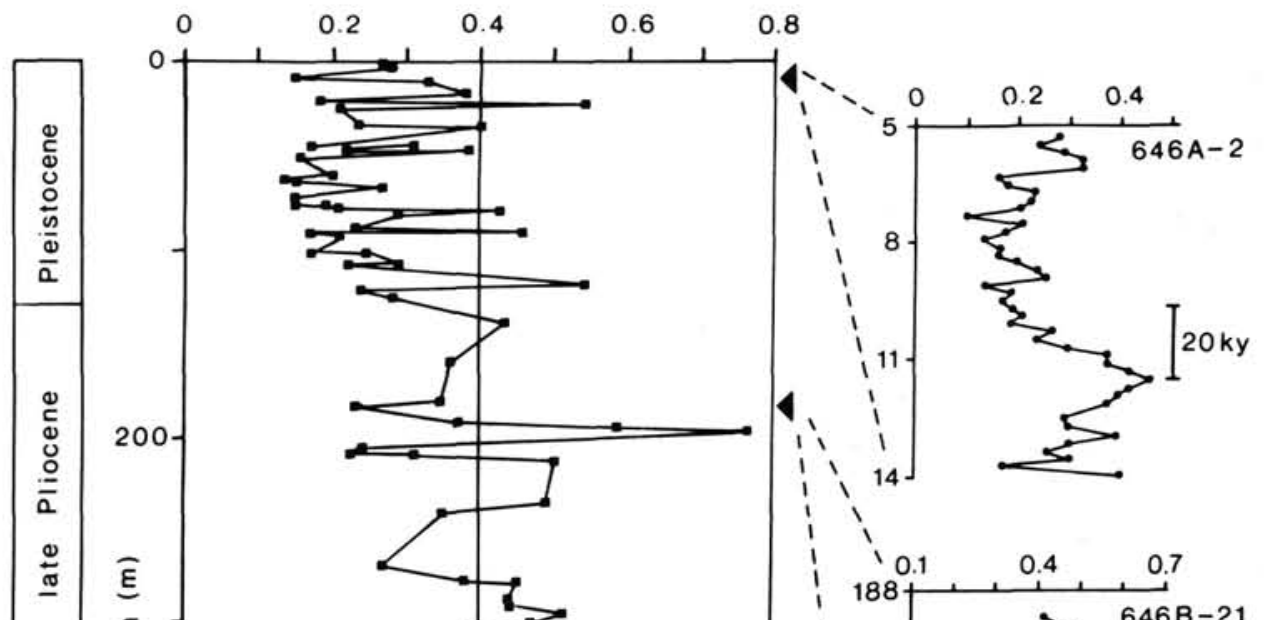

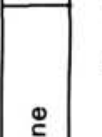

$\widehat{E}$
$\frac{5}{\vdots}$
$\frac{0}{0}$
$\vdots$

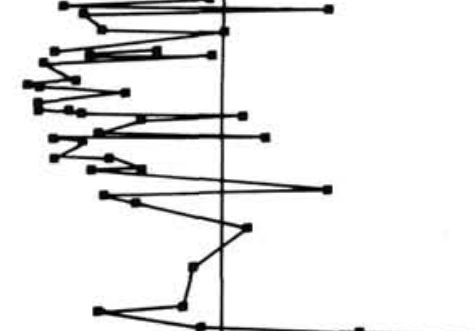

$200-$

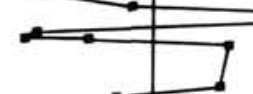

14

i

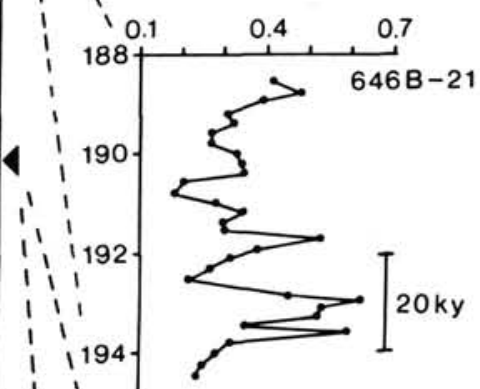

\}

$400-$
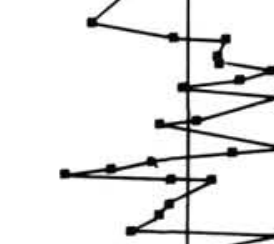

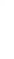
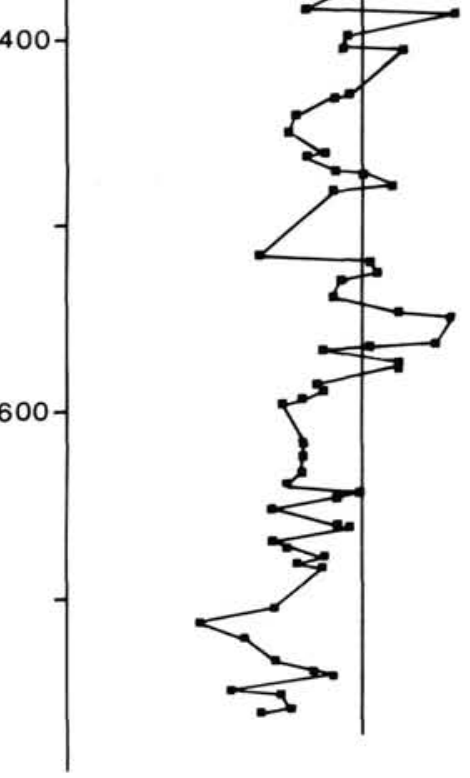

1

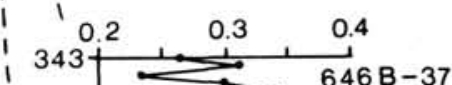

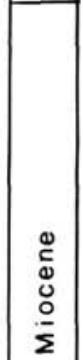
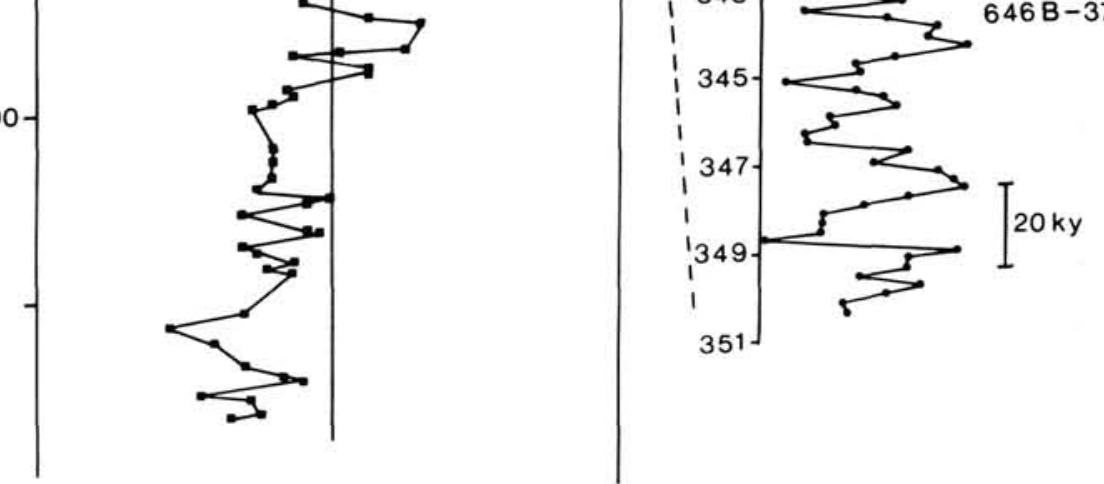

Figure 7. Total-organic-carbon contents at Site 646 (long-term and short-term records). 
647

TOC (\%)

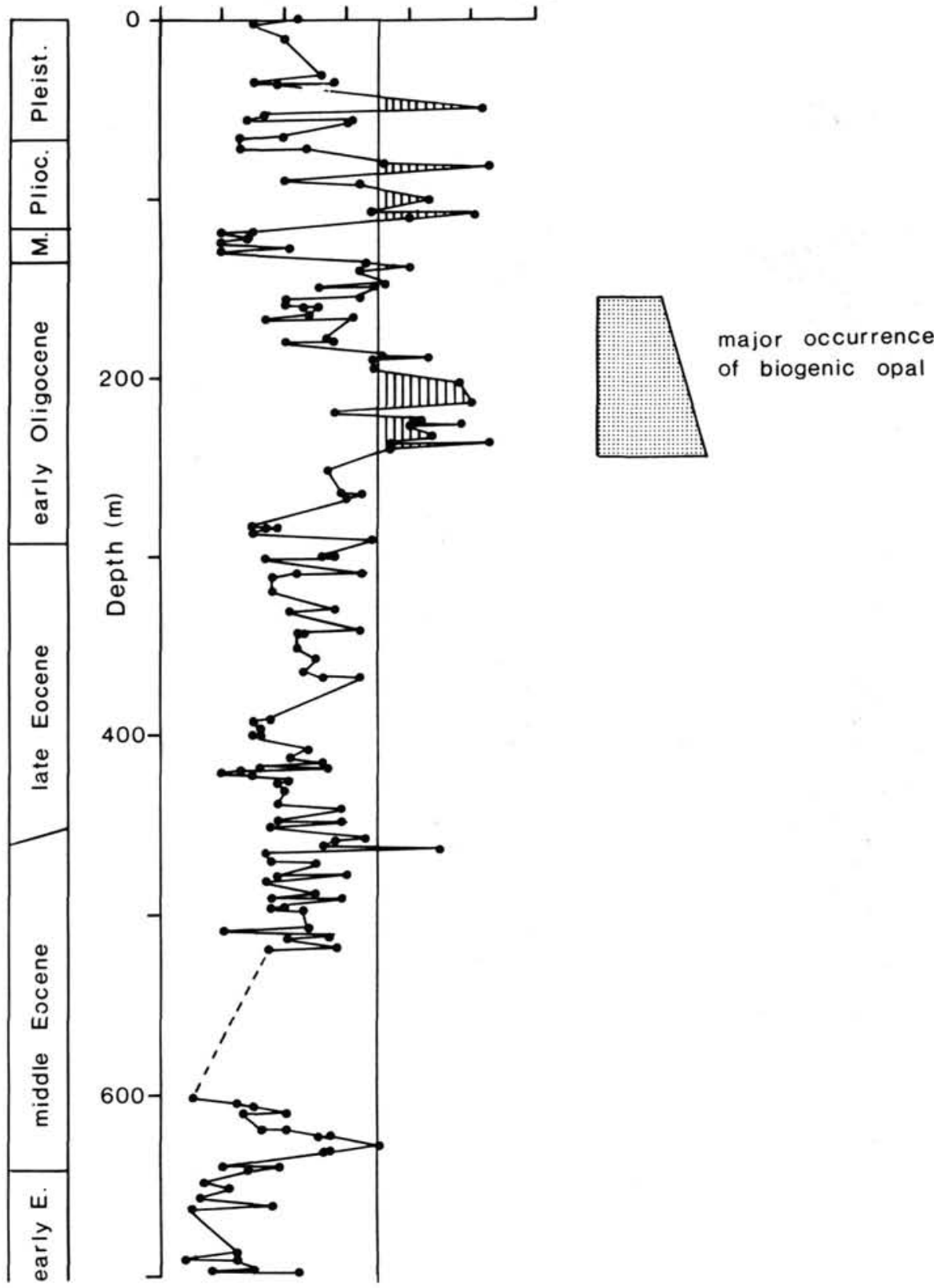

Figure 8. Total-organic-carbon contents at Site 647. The record includes data from Table 5 Nielsen et al. (this volume), and Bohrmann and Stein (this volume). Major occurrence of biogenic opal according to Bohrmann and Stein (this volume). 
645

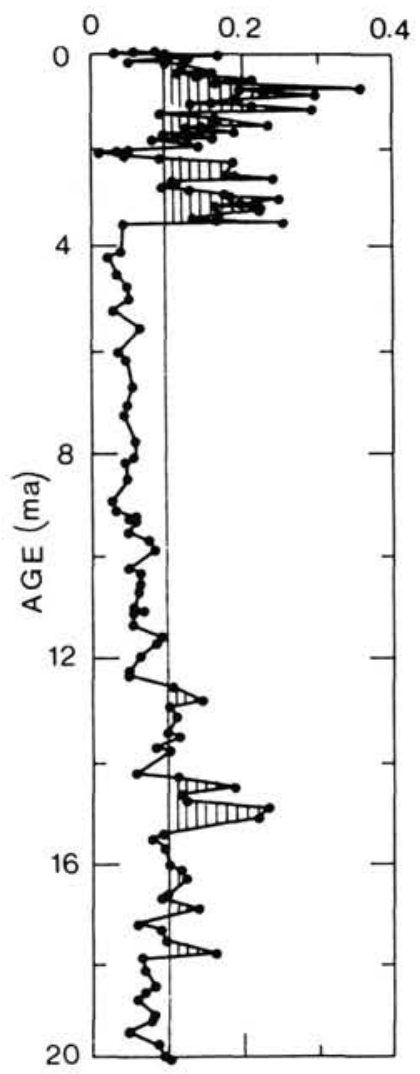

646

TOC $\left(\mathrm{gC} \cdot \mathrm{cm}^{-2} \cdot \mathrm{ky}^{-1}\right)$

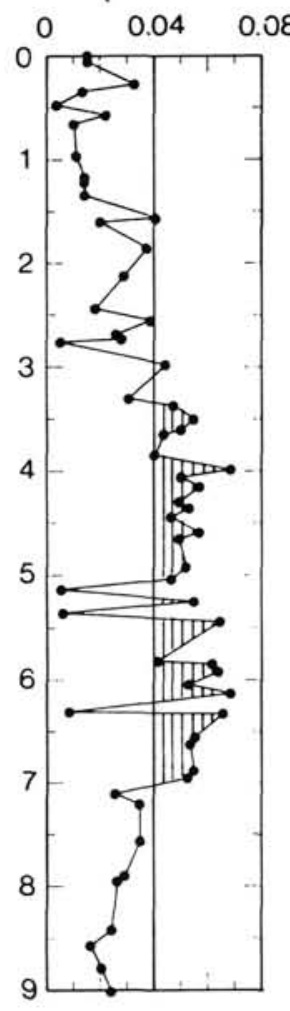

647

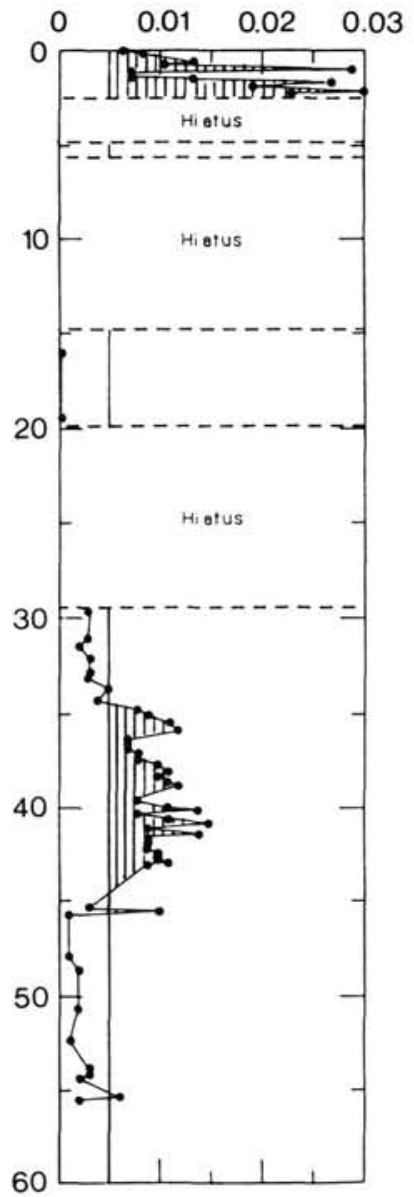

Figure 9. Accumulation rates of total organic carbon at Sites 645, 646, and 647 (Srivastava, Arthur, et al., 1987). 


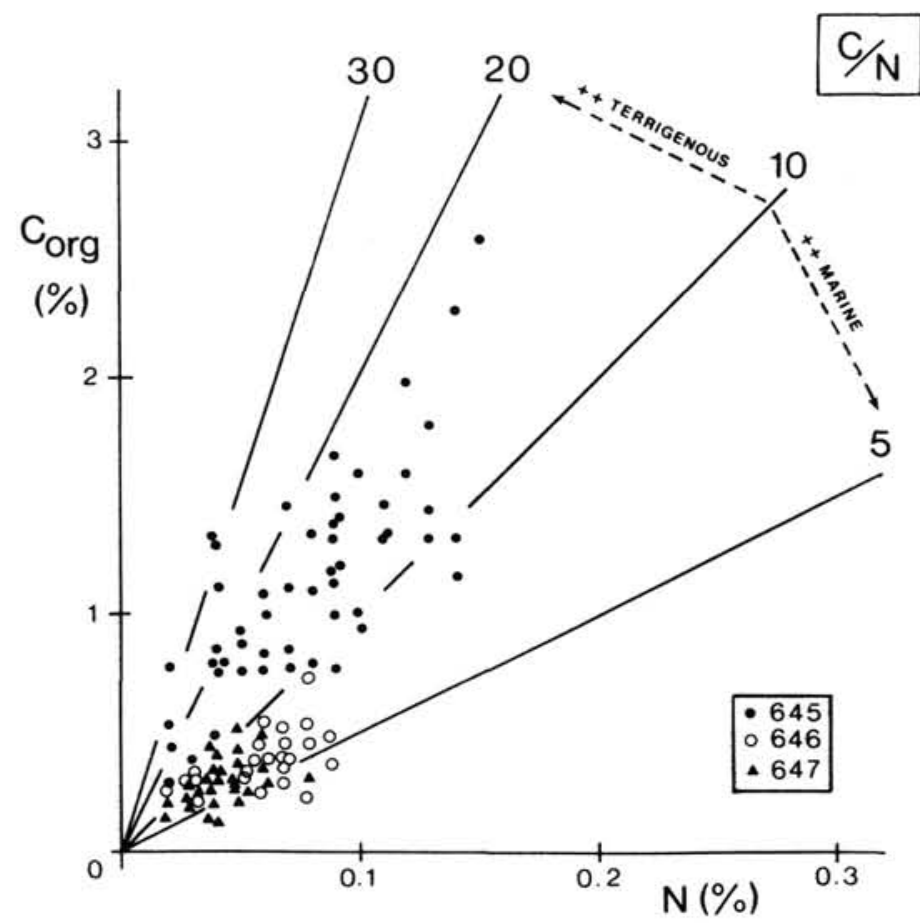

Figure 10. Total organic carbon vs. total nitrogen (in wt \%). Lines indicate $\mathrm{C} / \mathrm{N}$ ratios (i.e., 30, 20,10, and 5). Solid circles indicate data from Site 645 , open circles represent data from Site 646 , and solid triangles include data from Site 647.

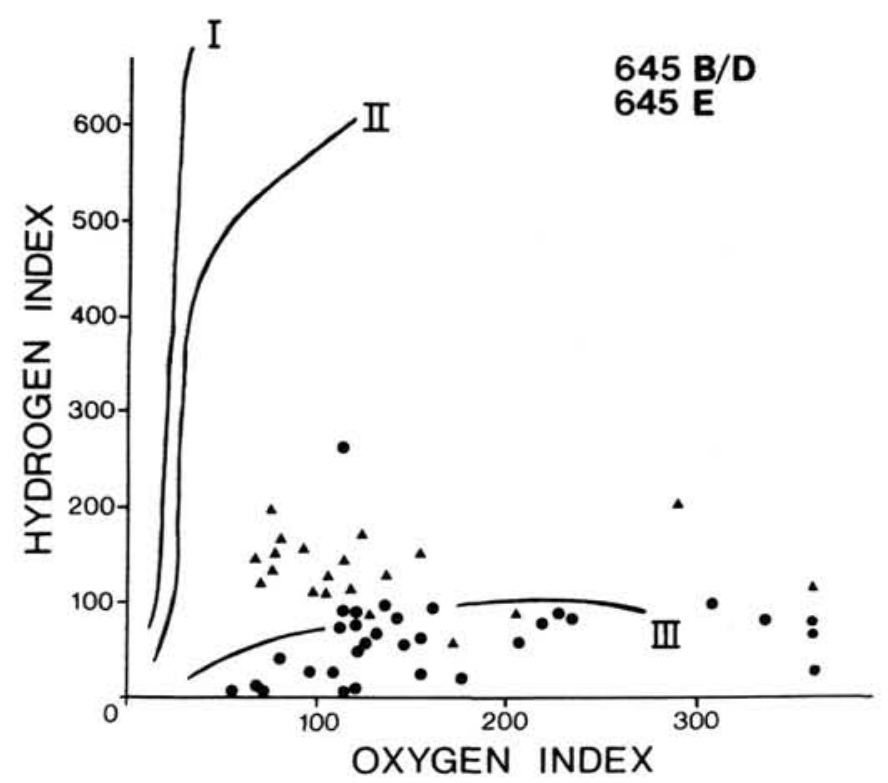

Figure 11. Results from Rock-Eval pyrolysis: hydrogen- and oxygen-index values (Shipboard data; Srivastava, Arthur, et al., 1987). 


\section{5}

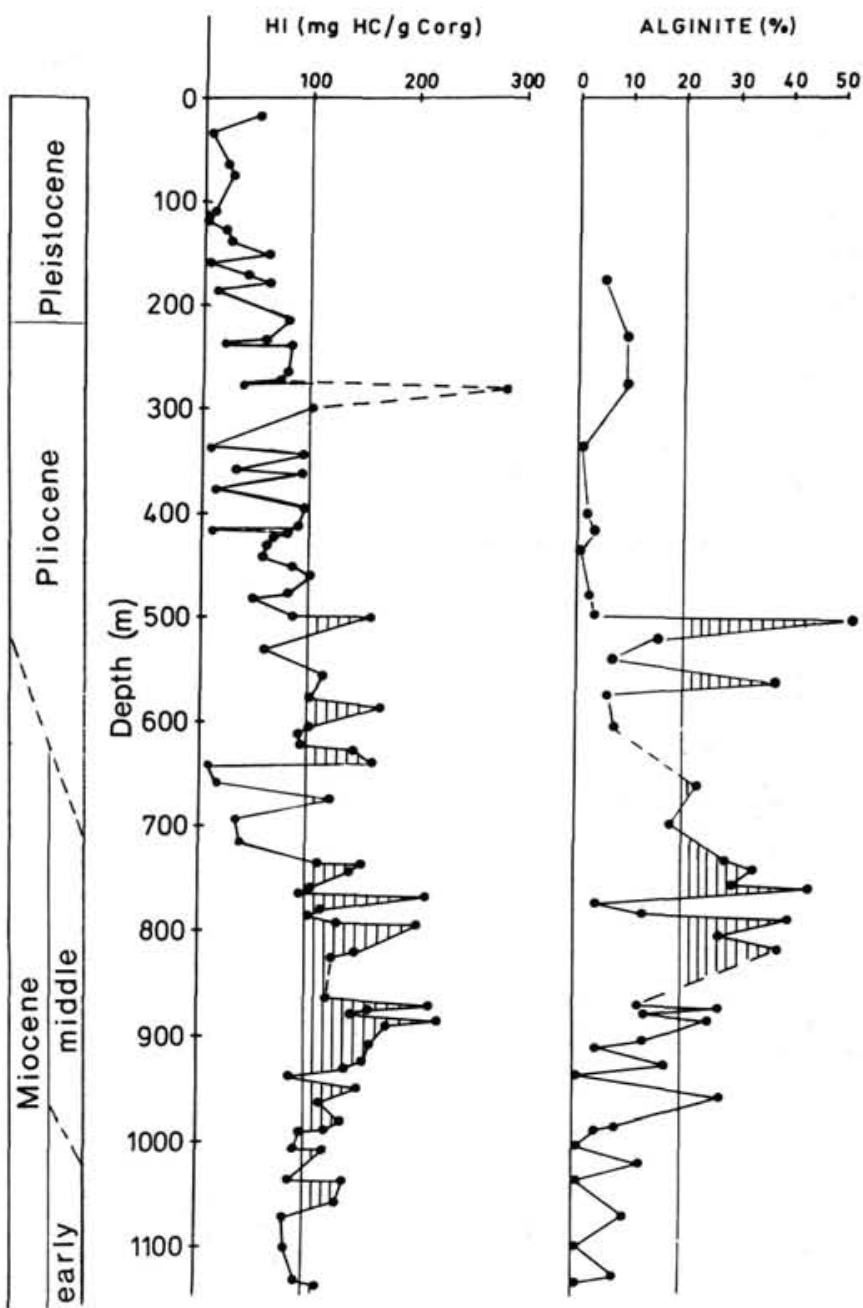

Figure 12. Hydrogen-index values and content of alginites from sediments at Site 645 .

Table 6. Results from Rock-Eval analyses for samples at Site 647 .

\begin{tabular}{cccc}
\hline $\begin{array}{c}\text { Core/section } \\
\text { interval }(\mathrm{cm})\end{array}$ & $\begin{array}{c}\text { TOC } \\
(\%)\end{array}$ & $\begin{array}{c}\mathrm{HI} \\
(\mathrm{mgHC} / \mathrm{gC})\end{array}$ & $\begin{array}{c}\mathrm{T}_{\max } \\
\left({ }^{\circ} \mathrm{C}\right)\end{array}$ \\
\hline $105-647 \mathrm{~A}-15-3,76$ & 0.29 & 72 & 435 \\
$16-1,98$ & 0.27 & 54 & 420 \\
$17-3,101$ & 0.14 & 43 & 422 \\
$19-3,88$ & 0.27 & 64 & 419 \\
$20-3,48$ & 0.27 & 81 & 419 \\
$23-1,114$ & 0.50 & 92 & 378 \\
$23-5,49$ & 0.28 & 57 & 414 \\
$24-1,130$ & 0.36 & 59 & 413 \\
$24-3,10$ & 0.41 & 64 & 419 \\
$24-3,119$ & 0.40 & 63 & 382 \\
\hline
\end{tabular}

$\mathrm{TOC}=$ total organic carbon; $\mathrm{HI}=$ hydrogen index

$\mathrm{T}_{\max }=$ temperature of maximum pyrolysis. 


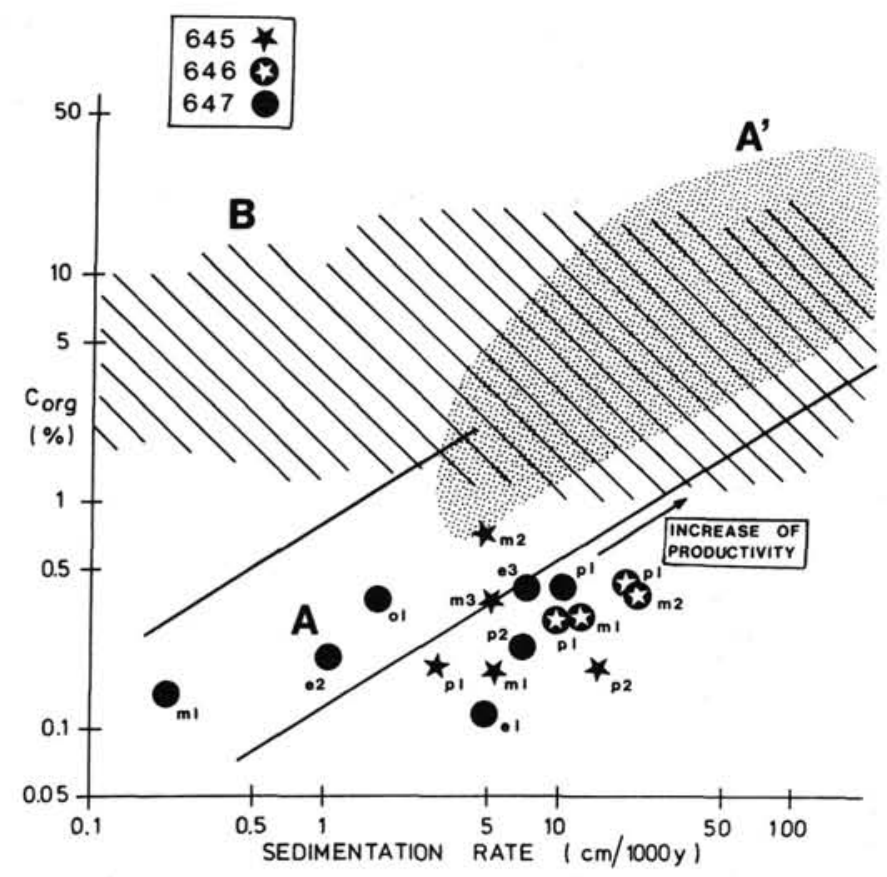

Figure 13. Correlation of (marine) organic-carbon values with sedimentation rates. The distinction between fields $A, A^{\prime}$, and $B$ is based on data from Holocene to Miocene sediments (Müller and Suess, 1979; Stein, 1986b). Field $A$ marks positive correlation of organic-carbon content with sedimentation rates under oxic deep-water conditions. Field $A^{\prime}$ indicates conditions of high oceanic productivity, characterized by high organiccarbon values and high sedimentation rates. In general, increased sedimentation rates coincide with increased productivity. Field $B$ indicates data derived from samples deposited under anoxic deep-water conditions. Sedimentation rates from Sites 645,646 , and 647 were corrected for compaction effects, following Stein (1986b). Solid stars indicate data from Site 645: p2 = late Pliocene/Pleistocene; p1 = latest Miocene/early Pliocene; $\mathrm{m} 3$ = late Miocene; $\mathrm{m} 2$ = middle Miocene; $\mathrm{m} 1=$ early Miocene. Open stars indicate data from Sites 646: p2 = late Pliocene/Pleistocene; $\mathrm{p} 1$ = early Pliocene; $\mathrm{m} 2$ = late Miocene (7-5.5 Ma); $\mathrm{m} 1$ = late Miocene (9-7 Ma). Solid circles indicate data from Site 647: p2 = Pleistocene; $\mathrm{p} 1$ = late Pliocene; $\mathrm{m} 1$ = Miocene; $\mathrm{ol}=$ (middle) Oligocene; $\mathrm{e} 3$ = late Eocene/early Oligocene; e2 = middle Eocene; el = early Eocene.

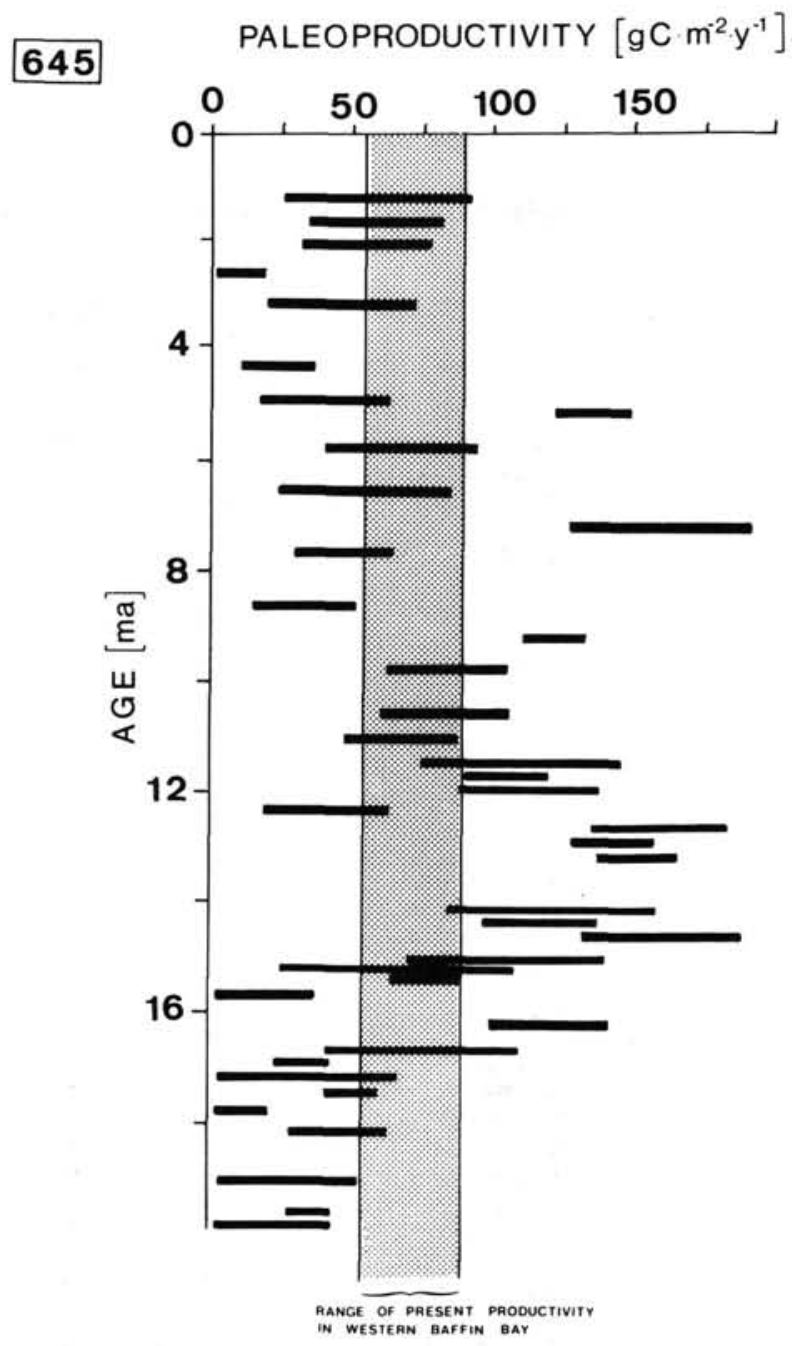

Figure 14. Estimated paleoproductivity from marine organic-carbon contents at Site 645, using Equation 4. Minimum and maximum productivity values (i.e., the width of the black bar) are based on minimum and maximum estimates of marine organic-carbon contents from kerogen microscopy. Physical-property data, paleowater depths, and sedimentation rates required for calculating paleoproductivity (see Equation 4) from Srivastava, Arthur, et al. (1987). Range of present productivity in western Baffin Bay from Romankevich (1984). 
Table 7. Estimated paleoproductivity values for different time intervals of sediments from Leg 105.

\begin{tabular}{|c|c|c|c|c|c|c|}
\hline $\begin{array}{l}\text { Age } \\
\text { (code) }\end{array}$ & $\begin{array}{l}\text { Sedimentation } \\
\text { rate } \\
(\mathrm{cm})\end{array}$ & $\begin{array}{l}\text { Porosity } \\
(\%)\end{array}$ & $\begin{array}{l}\text { Wet-bulk } \\
\text { density } \\
\left(\mathrm{g} / \mathrm{cm}^{3}\right)\end{array}$ & $\begin{array}{c}\text { Marine } \\
\text { organic carbon } \\
(\%)\end{array}$ & $\begin{array}{l}\text { Water } \\
\text { depth } \\
\text { (m) }\end{array}$ & $\begin{array}{l}\text { Paleoproductivity } \\
\left(\mathrm{gC} \cdot \mathrm{m}^{-2} \cdot \mathrm{y}^{-1}\right)\end{array}$ \\
\hline \multicolumn{7}{|l|}{ Site 645} \\
\hline $\begin{array}{l}\text { late Pliocene/ (p2) } \\
\text { Pleistocene }\end{array}$ & 13.5 & 46 & 2.02 & $\begin{array}{c}0.21 \\
(0.13)\end{array}$ & 2000 & $60-90$ \\
\hline $\begin{array}{l}\text { latest Miocene/ (p1) } \\
\text { early Pliocene }\end{array}$ & 2.8 & 49 & 1.92 & $\begin{array}{l}0.18 \\
(0.14)\end{array}$ & 1500 & $50-60$ \\
\hline late Miocene $(\mathrm{m} 3)$ & 4.8 & 45 & 2.02 & $\begin{array}{l}0.36 \\
0.18\end{array}$ & 1200 & $60-100$ \\
\hline middle Miocene $(\mathrm{m} 2)$ & 4.8 & 41 & 2.07 & $\begin{array}{l}0.7 \\
(0.40)\end{array}$ & 1000 & $100-150$ \\
\hline early Miocene $(\mathrm{ml})$ & 4.8 & 33 & 2.17 & $\begin{array}{c}0.17 \\
(0.13)\end{array}$ & 800 & $40-50$ \\
\hline \multicolumn{7}{|l|}{ Site 646} \\
\hline Pleistocene (p2) & 7.9 & 72 & 1.62 & 0.3 & 3450 & 95 \\
\hline late Pliocene (p2) & 7.9 & 67 & 1.67 & 0.3 & & 100 \\
\hline early Pliocene (p1) & 9.6 & 56 & 1.89 & 0.39 & & 150 \\
\hline $\begin{array}{l}\text { late Miocene }(\mathrm{m} 2) \\
(7-5.5 \mathrm{Ma})\end{array}$ & 9.6 & 46 & 2.11 & 0.37 & & 170 \\
\hline $\begin{array}{l}\text { late Miocene }(\mathrm{m} 1) \\
\quad(9-7 \mathrm{Ma})\end{array}$ & 4.9 & 44 & 2.14 & 0.3 & & 140 \\
\hline \multicolumn{7}{|l|}{ Site 647} \\
\hline Pleistocene (p2) & 4.6 & 63 & 1.72 & 0.22 & 3870 & 90 \\
\hline late Pliocene $(\mathrm{p} 1)$ & 4.6 & 52 & 1.94 & 0.39 & & 160 \\
\hline Miocene $(\mathrm{ml})$ & 0.2 & 81 & 1.43 & 0.16 & & 40 \\
\hline Oligocene (o1) & 1.6 & 78 & 1.46 & 0.4 & & 90 \\
\hline $\begin{array}{l}\text { late Eocene/ }(\mathrm{e} 3) \\
\text { earliest Oligocene }\end{array}$ & 3.6 & 54 & 1.9 & 0.4 & & 140 \\
\hline middle Eocene (e2) & 0.4 & 43 & 2.13 & 0.22 & $\begin{array}{l}2000- \\
3000\end{array}$ & 90 \\
\hline early Eocene (el) & 2.5 & 39 & 2.19 & 0.11 & & 70 \\
\hline
\end{tabular}

Note: Marine organic-carbon contents at Site 645 are from kerogen microscopy estimates (minimum and maximum values). At Sites 646 and 647 , we assumed that total organic carbon recorded at both sites is mostly of marine origin, which is supported by $\mathrm{C} / \mathrm{N}$ ratios. Physical property data and sedimentation rates from Srivastava, Arthur, et al., 1987. 